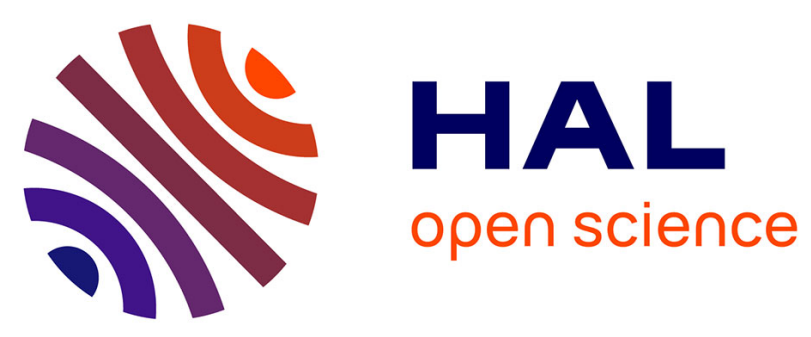

\title{
Deduction and computation
}

\author{
Gérard Huet
}

\section{To cite this version:}

Gérard Huet. Deduction and computation. RR-0513, INRIA. 1986. inria-00076041

\section{HAL Id: inria-00076041 \\ https://hal.inria.fr/inria-00076041}

Submitted on 24 May 2006

HAL is a multi-disciplinary open access archive for the deposit and dissemination of scientific research documents, whether they are published or not. The documents may come from teaching and research institutions in France or abroad, or from public or private research centers.
L'archive ouverte pluridisciplinaire HAL, est destinée au dépôt et à la diffusion de documents scientifiques de niveau recherche, publiés ou non, émanant des établissements d'enseignement et de recherche français ou étrangers, des laboratoires publics ou privés. 


\section{Rapports de Recherche}

$\mathrm{N}^{\mathrm{o}} 513$

\section{DEDUCTION \\ AND \\ COMPUTATION}

Gérard HUET

H.

W

-7.3.

3:07

6.

(6)

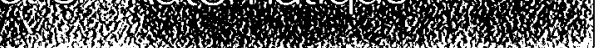

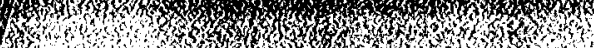
15)

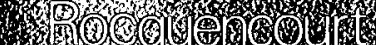

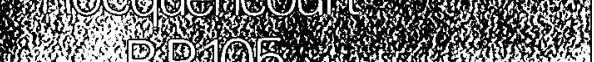
1)

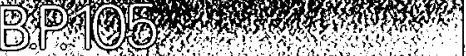
$\%$

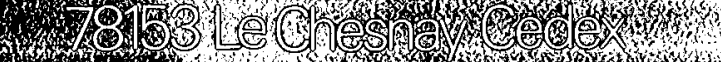

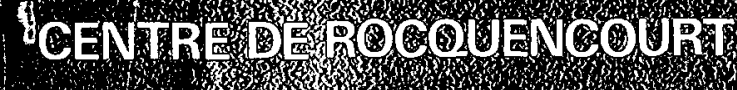




\section{Decuetion and Computation}

\section{Gérand fuct}

\section{Résumé}

Nons présentons dans un carle commun les concopts syntaxirues eseontiels à une théorie unifiéc des preuves et des calculs.

\section{Abstract}

We present in a unified framework the basic syntautic notions of deduction and computation.

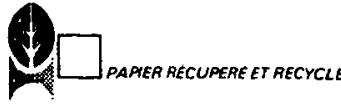




\title{
Deduction and Computation
}

\author{
Gérard Huet
}

We present in a unified framework the basic syntactic notions of deduction and computation.

\section{Terms and types}

\subsection{General notations}

We assume known elementary set theory and algebra. $\mathcal{N}$ is the set $\{0,1, \ldots\}$ of naturil numbers, $N_{+}$the set of positive natural unmbers. We shall identify the natural $n$ with the set $\{0, \ldots, n-1\}$, and thus 0 is also the emply set $\emptyset$. Every finite set $S$ is isomorphic to $n$, with $n$ the cardinal of $S$, denoted $n=|S|$. If $A$ and $B$ are sets, we write $A \rightarrow B$, or sometimes $B^{A}$, for the set of functions with domain $A$ and codonain $B$.

\subsection{Languages, concrete syntax}

Let $\Sigma$ be a finite alphabet. A string $u$ of length $n$ is a function in $n \rightarrow \Sigma$. The sct of all strings over $\Sigma$ is

$$
\Sigma^{*}=\bigcup_{n \in \mathcal{N}} \Sigma^{n}
$$

We write $|u|$ for the length $n$ of $u$. We write $u_{i}$ for $u(i-1)$, when $i \leq n$. The null string, unique element of $\Sigma^{0}$, is denoted $\Lambda$. The unit string mapping 1 to $a \in \Sigma$ is denoted ' $a$ '. The concatenation of strings $u$ and $v$, defined in the usual fashion, is denoted $u^{-} v$, and when there is no ambiguity we write e.g. ' $a b c^{\prime}$ for ' $a c^{-} b^{\prime}-' c^{\prime}$. When $u \in \Sigma^{*}$ and $a \in \Sigma$, we write $u \cdot a$ for $u^{-}{ }^{\prime} a^{\prime}$. We define an ordering $\leq$ on $\Sigma^{*}$, called the prefix ordering, by

$$
u \leq v \Leftrightarrow \exists w \quad v=u^{-} w
$$

If $u \leq v$, the residual $w$ is unique, and we write $w=v / u$. We say that occurrences $u$ and $v$ are disjoint, and we write $u \mid v$, iff $u$ and $v$ are unrelated by the partial ordering $\leq$. Finally we let $u<v$ iff $u \leq v$ with $u \neq v$.

The set $\Sigma^{*}$ has the structure of a monoïd, that is:

$$
\begin{gathered}
\text { Ass: }(u-v)-w=u-(v-w) \\
I d L: \Lambda-u=u \\
I d R: u-\Lambda=u
\end{gathered}
$$

Actuially, $\Sigma^{*}$ is the free monoïd generated by $\Sigma$.

\section{Examples.}

1. $\Sigma=0$. We get $\Sigma^{*}=1$.

2. $\Sigma=1$. We get $\Sigma^{*}=\mathcal{N}$. Strings are here natural numbers in unary notation, and concatenation corresponds to addition.

3. $\Sigma=2=\{0,1\}$ (the Booleans). The set $\Sigma^{*}$ is the set of all biniry words.

4. $\Sigma=\mathcal{N}_{+}$. We call the dements of $\Sigma^{*}$ occurrences. When $u=w \cdot r n$ and $v=w \cdot n$, with $m<n$, we say that $u$ is left of $v$, and write $u<L v$. 


\subsection{Terms: abstract syntax}

We first define a tree domain as a subset, $D$ of $\mathcal{N}_{f}^{*}$ closed under $<$ and $<_{L}$ :

$$
\begin{gathered}
u \in D \wedge v<u \Rightarrow v \in D \\
u \in D \wedge v<_{L} u \Rightarrow v \in D .
\end{gathered}
$$

We say that $M$ is a $\Sigma$-tree iff $M \in D \rightarrow \Sigma$, for some tree domain $D$. We write $D=D(M)$, and we say that $D$ is the set of occurrences in $M$. $M$ is said to be finite whenever $D$ is.

We shall now use occurrences to designate nodes of a trec, and the subtrec starting at that node. If $u \in D(M)$, we define the $\Sigma$-trec $M / u$ as manping occurrence $v$ to $M\left(u^{-} v\right)$. We say that $M / u$ is the sub-tree of $M$ at occurrence $u$. If $N$ is also a $\Sigma$-tree, we define the gratt $M[u+N]$ as the $\Sigma$-tree mapping $v$ to $N(w)$ whenever $v=u^{-} w$ with $w \in D(N)$, and to $M(v)$ if $v \in D(M)$ and not $u \leq v$.

We need one anxiliary notion, that of width of a tree. If $M \in \Sigma^{*}$, we define the (top) width of $M$ as

$$
\|M\|=\max \left\{\left.n\right|^{\prime} n^{\imath} \in D(M)\right\}
$$

We shall now consider $\Sigma$ a graded alphabet, that is given with an arity function $\alpha$ in $\Sigma \rightarrow \mathcal{N}$. We then say that $M$ is a $\Sigma$-term iff $M$ is a $\Sigma$-tree verifying the supplementary consistency condition:

$$
\forall u \in D(M)\|M / u\|=\alpha(M(u))
$$

That is, every subtree of $M$ is of the form $F\left(M_{1}, M_{2}, \ldots, M_{n}\right)$, with $n=\alpha(F)$. We write $T(\Sigma)$ for the set of $\Sigma$-terms. If $M_{1}, M_{2}, \ldots M_{n} \in T(\Sigma)$ and $F \in \Sigma$, with $\alpha(F)=n$, then $M=F\left(M_{1}, M_{2}, \ldots M_{n}\right)$ is easily defined as a $\Sigma$-term. This gives $T(\Sigma)$ the structure of a $\Sigma$-algebri. Since conversely the decomposition of $M$ is uniquely determined, we call $T(\Sigma)$ the completely free $\Sigma$-algebra.

\section{Example}

With $\Sigma=\{+, S, 0\}, \alpha(+)=2, \alpha(S)=1, \alpha(0)=0$, the following structure represents a $\Sigma$-term:

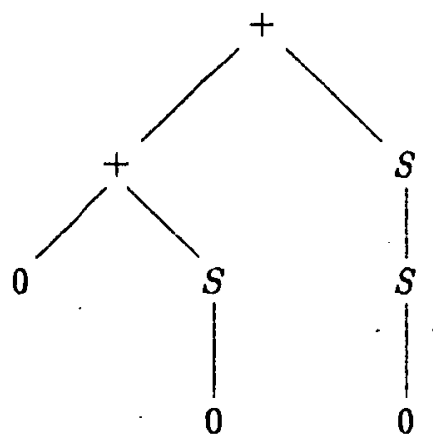

The following proposition is easy to prove by induction. All occurrences are supposed to be universally quantified in the relevant tree domain.

Proposition 1.

$$
\begin{gathered}
\text { Embedding: } M[u \leftarrow N] /\left(u^{-} v\right)=N / v \\
\text { Associativity: } M[u \leftarrow N]\left[u^{-} v-P\right]=M[u \leftarrow N[v \leftarrow P]]
\end{gathered}
$$




$$
\begin{gathered}
\text { Persistence }: M[u \leftarrow N] / v=M / v \quad(u \mid v) \\
\text { Commatativity : } M[u \leftarrow N][v \leftarrow P]=M[v \leftarrow P][u \leftarrow N] \quad(u \mid v) \\
\text { Distributivity }: M[u \leftarrow N] / v=(M / v)[u / v \leftarrow N] \quad(v \leq u) \\
\text { Dominance : } M[u \leftarrow N][v \leftarrow P]=M[v \leftarrow P] \quad(v \leq u)
\end{gathered}
$$

We define the length $|M|$ of a (finite) term $M$ recursively by:

$$
\left|F^{\prime}\left(M_{1}, \ldots, M_{2}\right)\right|=1+\sum_{i=1}^{n}\left|M_{i}\right|
$$

\subsection{Parsing}

It is woll-known that the term in the cxample above can be represented unambiguously as a $\Sigma$-string, for instance in prefix polish notation, that is here: $++0 S 0 S S 0$. This result is not very interesting: such strings are neither good notations for humans, nor good reprosentations for computers, since the graft operation necessitates unnecessary copying. Wo shall discuss later good machine representations, using binary graphs. As far as human readibility is concened, we assume known parsing technicues. This permits to represent terms, on an extended alphabet with parentheses and commas, which is closer to standard mathematical practice. Mlso, infix notation and indentation permit to keep in the string some of the tree structure more apparent. We shall not make explicit the exact representation grammar, and allow ourselves to writc freely for instance $(0+S(0))+S(S(0))$. Note that we avoid explicit quotes as well, which permits us to mix frcely meta-variables with object structures, like in $S(M)$, where $M$ is a meta-variable denoting a $\Sigma$-term.

\subsection{Terms with variables, substitution}

The idea is to internalize the notation $S(M)$ above as a term $S(x)$ over an extended alphabet containing special symbols of arity 0 called viriables.

Let $V$ be a clenumerable set disjoint from $\Sigma$. We define the set of terms with variables, $T(\Sigma, V)$, in exactly the same way as $T(\Sigma \cup V)$, extending the arity function so that $\alpha(x)=0$ for cvery $x$ in $V$. The only difference between the variables and the constants (symbol of arity 0 ) is that a constant has an existential import: it denotes a value in the domain we are modelling with our term language, whereas a variable denotes a term. The difference is important only when there are no constants in $\Sigma$, since then $T^{\prime}(\Sigma)$ is empty.

All of the notions defined for terms extend to terms with variables. We define the set $V(M)$ of variables occurring in $M$ as:

$$
V(M)=\{x \in V \mid \exists u \in D(M) \quad M(u)=x\}
$$

and we define the number of distinct variables in $M$ as $\nu(M)=|V(M)|$.

We shall now formalize the notion of substitution of terms for variables in a term containing variables. From now on, the sets $\Sigma$ and $V$ are fixed, and we use $T$ to denote $T(\Sigma, V)$. A substitution $\sigma$ is a function in $V \rightarrow T$, identity almost evcrywhere. That is, the set $D(\sigma)=\{x \in V \mid \sigma(x) \neq x\}$ is finite. We call it the domain of $\sigma$. Substitutions are extended to morphisinns over $T$ hy

$$
\sigma\left(F\left(M_{1}, \ldots, M_{n}\right)\right)=F\left(\sigma\left(M_{1}\right), \ldots, \sigma\left(M_{n}\right)\right)
$$

Bijective substitutions are called permutations. When $U \subseteq V$, we write $\sigma_{U}$ for the restriction of substitution $\sigma$ to $U$. It is casy to show that, for all $\sigma, M$ and $U$ :

$$
V(M) \subseteq U \Rightarrow \sigma(M)=\sigma_{U}(M)
$$


Alteruatively, we cian define the replacenene $M[x-N]$ as

$$
M\left[u_{1} \leftarrow N\right] \ldots\left[u_{n}+N\right]
$$

where $\left\{u_{1}, \ldots, u_{n}\right\}==\{u \mid M(u)=x\}$ and then

$$
\sigma(M)=M[x+\sigma(x) \mid x \in V(M)]
$$

with an obvious notation.

We now define the quasi-ordering $\leq$ of matching in $T$ by:

$$
M \leq N \quad \Leftrightarrow \quad \exists \sigma \quad N=\sigma(M)
$$

It is casy to show that if such a $\sigma$ exists, $\sigma_{V(M)}$ is unique. Wo shall call it the match of $N$ by $M$, and deriote it by $N / M$.

We define $M \equiv N \Leftrightarrow M \leq N \wedge N \leq M$. When $M \equiv N$, we say that $M$ and $N$ are isomorphic. This is equivalent to say that $M=\sigma(N)$ for some permutation $\sigma$. Note that $M \equiv N$ implics $|M|=|N|$. Finally, we define

$$
M>N \Leftrightarrow N \leq M \wedge \neg M \leq N .
$$

Proposition. $>$ is a wcll-ordering on $T$.

Proof. We show that $M>N$ implies $\mu(M)>\mu(N)$, with $\mu(M)=|M|-\nu(M)$.

Let $\varphi$ be any bijection between $T \times T$ and $V$. We define a binary operation $\cap$ in $T$ by:

$$
\begin{gathered}
F\left(M_{1}, \ldots, M_{n}\right) \cap F\left(N_{1}, \ldots, N_{n}\right)=F\left(M_{1} \cap N_{1}, \ldots, M_{n} \cap N_{n}\right) \\
M \cap N=\varphi(M, N) \text { in all other cases. }
\end{gathered}
$$

$M \cap N$ is uniquely determined from $\varphi$ and, for distinct $\varphi^{\prime} s$, is unique up to $\equiv$.

Proposition. $M \cap N$ is a g.l.b. of $M$ and $N$ under the match quasi-ordering.

Let $\mathbf{T}$ be the quotient set $T / \equiv$, completed with a maximum element $T$. Frum the propositions above we conclinde:

Theorem. $\mathbf{T}$ is a complete lattice.

Corollary. If two tcrms $M$ and $N$ have an upper bound, i.e. a common instance $\sigma(M)==\sigma^{\prime}(N)$, they have a l.u.b. $M \cup N$, which is a most general such instance: $\sigma=\sigma_{0} ; \tau, \sigma^{\prime}=\sigma_{0}^{\prime} ; \tau$. The term $M \cup N$ is unique modulo $\equiv$ and may be found by the unification algorithm.

Proposition.

$$
\begin{gathered}
D(\sigma(M))=D(M) \cup \bigcup_{\{u \mid M(u) \in V\}}\{u-v \mid v \in D(\sigma(M(u)))\} \\
\forall u \in D(M) M(u) \in V \Rightarrow \sigma(M) / u-v=\sigma(M(u)) / v(v \in D(\sigma(M(u)))) \\
M(u) \in \Sigma \Rightarrow \sigma(M) / u=\sigma(M / u) \\
\sigma(M)[u \leftarrow \sigma(N)]=\sigma(M[u \leftarrow N])
\end{gathered}
$$




\subsection{Graph representations, dags}

We represent trees by binary graphs of adr pairs. An anly consists in one tag bit, and one byte ficld interpreted cither as an address in the graph menory, or as a natural number. In this last case, the uatural 0 is reserved for nil, the empty list of trees. Symbols from $\Sigma$ are coded as positive naturals. If tree $M$ is represented at the graph address $a d r 1$ and the list $L$ is represented at address $a d r 2$, then the list $M \cdot L$ is represented by the graph node $(M \cdot L)$. Finally, the tree $F^{\prime}(L)$ is represented by $(F \cdot L)$.

This is the standard way of representing trees and lists in the language LISP. A precise description of the memory allocation implementation of such schemes is beyond the scope of these notes.

Terms are of course represented as trees. A global table holds the arity function. There are several possibilities for the representation of variables. They may be represented as symbols. But then the scope structure must be computed by an algorithm, rather than being implicit in the structure. Also a global scanning of the term is necessary to determine its sct of variables, and substitution involves copying of the substituted term. For these reasons, variables are oftcr represented rather as integer offsets in stacks of bindings. Such "structure sharing" representations are now standiard for PROLOG implementations.

A precisc account of the various representations schemes for term structures, and of the accompanying algorithms, is out of the scope of these notes. It sllould be born in mind that the crucial problem is momory utilization: the trarle-off between copying and sharing is often the deciding factor for an implementation. Languages with garbage-collected structures, such as LISP, are ideal for programming "quick and dirty" prototypes. But serious implementation efforts should aim at good algorithmic performance on realistic size applications.

The crucial algorithms in formula and proof manipulation are matching, unification, substitution and grafting. First-order unification has been specially well studied. A linear algorithm is known [122], but in practice quasi-linear algorithms based on congruence classes operations arc preferred $[00,100]$. Furthermore, these algorithms extend without modification to unification of infinite rational terms represented by finite graphs [64].

Implementation methods may be partitioned into two families. Some depend on logical properties (e.g. sharing subterms in dags arising from substitution to a term containing several occurrences of the same variable). Some are purely statistical (e.g. sharing structures globally through hash-coding techniques). Particular applications require a careful analysis of the optimal trade-off between logical and statistical techniques.

There is no comprehensive survey on implementation issues. Some partial aspects are described in $[8,141,101,90,164,150,115,40,1,32,42,19,45,145,160]$.

\section{Inference rules}

We shall now study inference systems, defined by inference rules. The general form of an inference rule is:

$$
I R: \frac{P_{1} P_{2} \ldots P_{n}}{Q}
$$

where the $P_{i}$ 's and $Q$ are propositions belonging to some formal languagc. We shall here regard these propositions as types, and the inference rule as the description of the signature of $I R$ considered as a typed operator. More precisely, $I R$ has arity $n, P_{i}$ is the type of its $i$-th argument, and $Q$ is the 
type of its result. Well-typed terms composed of inference operators are called the proofs defined by the inforence system. Let us now exiunine a few familiar inferonce systems.

\subsection{The trivial homogeneous case: Arities}

A graded alphabet $\Sigma$ may be considered as the simplest inference systems, where types are reduced to arities. L.e., the set of propositions is 1 , and an operator $F$ of arity $n$ is an inference rule

$$
F: \frac{00 \ldots 0}{0}
$$

(with $n$ zcro's in the munerator). A $\Sigma$-proof corresponds to our $\Sigma$-terms above.

\subsection{Finite systems of types: Sorts}

The next level of inference systems consist in choosing a finite set $S$ of elementary propositions, usually called sorts. For instance, with $S=\{$ int, bool $\}$, and $\Sigma$ delincd by:

$$
0: \text { int } S: \text { int } \rightarrow \text { int true : bool false: bool if : bool, int } \rightarrow \text { int }
$$

where we use the alternative syntax $P_{1}, \ldots, P_{n} \rightarrow Q$ for an inference rule, the term if $($ true, $0, S(0))$ is of sort int, i.c. it is a proof of proposition int.

As another example, consider the puzzle "Missionaries and Cannibals". We call configuration any triple $\langle b, m, c\rangle \in 2 \times 4 \times 4$. The boolean $b$ indicates the position of the boat, $m$ (resp. $c$ ) is the number of missionaries (resp. cannibals) on the left bank. The set of states $S$ is the set of legal configurations, that obey the condition

$$
P(m, c) \equiv m=c \text { or } m=0 \text { or } m=3
$$

There arc thus 10 distinct states or sorts. The rules of inference comprise first a constant denoting the starting configuration:

$$
s_{0}:\langle 0,3,3\rangle
$$

then the transitions carrying $p$ missionaries and $q$ cannibals from left to right:

$$
L_{m, c, p, q}:\langle 0, m, c\rangle \rightarrow\langle 1, m-p, c-q\rangle \quad(m \geq p, c \geq q, P(m, c), P(m-p, c-q), 1 \leq p+q \leq 2)
$$

and finally the transitions $R_{m, c, p, q}$, which are inverses of $L_{m, c, p, q}$. The garne consists in finding a proof of $\langle 1,0,0\rangle$.

This simple example of a finite group of transformations applies to more complex tasks, such as Rubik's cube. All statte transition systcrns can be described in a similar fashion. Examples of such proofs are parse-trees of regular grammars, where the inference rules signatures correspond to a finite antomaton transition graph. Slightly more complicated formalisms allow subsorts, i.e. containment relationships between the sorts, i.c. implications between the elcunentiary propositions. These systems reduce to simple sorts by considering dummy transitions curresponding to the implicit coercions. 


\subsection{Types as terms: standard proof trees}

We shall here describe our types as terms formed over an alphabet, $\Phi$ of type operatoss, which we shall call functors. For the monent, we shall assume that we have just, one category of such propositions, i.e. the functors have just an arity. The alphabet $\Sigma$ of inference cules determines the legal proof trees.

\section{Example. Combinatory logic.}

We take as functors a set $\Phi$ of constants $\Phi_{0}$, plus a binary operator $\rightarrow$, which we shall write in infix notation. We call functionality a term in $T(\Phi)$. We have three families of rules in $\Sigma$. Th the following, the meta-variables $A, B, C$ denote arbitrany functionalitics. The operators of the $K$ and $S$ families are of arity 0 , the operators of the $A p p$ family are binary.

$$
\begin{gathered}
K_{A, B}: A \rightarrow(B \rightarrow A) \\
S_{A, B, C}:(A \rightarrow(B \rightarrow C)) \rightarrow((A \rightarrow B) \rightarrow(A \rightarrow C)) \\
A_{p p_{A, B}:} \frac{A \rightarrow B A}{B}
\end{gathered}
$$

Here is an example of a proof. Let $A$ and $B$ be any functionalities, $C=B \rightarrow A, D=A \rightarrow C$, $E=A \rightarrow A, F=A \rightarrow(C \rightarrow A), G=D \rightarrow E$. The term

$$
\operatorname{App}_{D, E}\left(A p_{F, G}\left(S_{A, C, A}, K_{A, C}\right), K_{A, B}\right)
$$

has type $E$, i.c. it gives a proof of the proposition $A \rightarrow A$.

We express formally that proof $M$ proves proposition $P$ in the inference system $\Sigma$ as: $\Sigma F=M$ : $P$. That is, we think of a theorem as the type of its proof tree. Proof-checking is identified with type-checking. Here this is a simple consistency check; that is, if operator $F$ is declared in $\Sigma$ as: $F: P_{1}, \ldots, P_{n} \rightarrow Q$ and if $\Sigma \vDash M_{i}: P_{i}$ for $1 \leq i \leq n$, then $\Sigma \vDash F\left(M_{1}, \ldots, M_{n}\right): Q$.

\subsection{Polymorphism: Rule schemas}

This next level of generality consists in authorizing variables in the propositional terms. This is very natural, since it internalizes the meta-variables used to index families of inference rules as propositional variables. The rules of inference become thus polymorphic operators, whose types are expressions containing free variables. This is the traditional notion of schematic inference rule from mathematical logic.

Example. The example from the previous section is more naturally expressed in this polymorphic formalisin. We replace the sct $\Phi_{0}$ by a set of variables $V$, and now we have just 3 mules of inference: $K, S$ and App. The types can be completely dispensed with, since a well typed term possesses a most gencral type, called its principal type. For instance, in the example above, the proof $\operatorname{App}(A p p(S, K), K)$ has a principal type $A \rightarrow A$, with $A \in V$. This term is ustally written $I=S K K$ in combinatory logic, where the concrete syntax convention is to write combinator strings to represent secguences of applications associated to the left.

The notion of principal type, first discovered by findley in the combinatory logic context, and independently by Milno: for ML type-checking [111], is actually completely general: 
Theorem. Iset $\Sigma$ be any siguature of polymorphic operaters over a functor sigmature $\Phi$. Let $M$ bo a legal proof tem. Then $M$ possesses a principal type $\tau \in T(\Phi, V)$. That is, $\Sigma \vDash M: \tau$, and for all $\tau^{\prime} \in T(\Phi, V), \Sigma \vDash M: \tau^{\prime}$ implies $\tau \leq \tau^{\prime}$.

Proof. This is an casy application of the unification theorem.

By now we have developed cuongh formalism to make scuse out of our "propositions as types" paradigm. Actually, the example we have discussed above is the fragment of propositional logic known as "minimal logic". When regarding the functor $\rightarrow$ as (intuitionistic) iujplication, and App as the usual inference rule of Modus ponens, $K$ and $S$ are the two axioms of minimal logic presented as a Ililbert calculns. Combinatory logic is thrs the calculus of proofs in minimal logic [37].

Actually combinators don't just have a type, they have a value. They can be defincd with definition equations in terms of application. Using the concrete syntax mentioned above, we get for instance $K$ and $S$ defined by the following equations:

$$
\begin{gathered}
\text { Def } f_{K}: K x y=x \\
\text { Def }_{S}: S x y z=x z(y z) .
\end{gathered}
$$

Exercice. Verify that the two equations above, when seen as unification constraints, define the expected principal types for $K$ and $S$.

This point of view of considering equality axiomatizations of the proof structures corresponds to what the proof-theorists call cut climination. That is, the two equations above can be used as rewrite rules in order to eliminate redundancies corresponding to useless detours in the proofs. We shall develop more completely this point of view of computation as proof normalization in section 4.4 below.

The current formalism of inference rules typed by terms with variables corresponds to proof theory's intuitionistic sccuents, and to automated reasoning's Horn clauses. For instance, a PROLOG [24] interpreter may be seen in this framework as a proof synthesis method. Given an alphabet $\Sigma$ of polymorphic inference rules (asually called defnite clauses), and a proposition $\tau$ over functor alphabet $\Phi$, it returns a proof term $M$ such that $M$ is a legal $\Sigma$-proof term of principal type $\tau^{\prime}$ instance of $\tau$ :

$$
\Sigma \models M: \tau^{\prime} \geq \tau \text {. }
$$

With $\sigma=\tau^{\prime} / \tau$, we say that $\sigma$ is a PROLOG answer to the query $\tau$. Of course this explanation is incomplete; we have to explain that PROLOG finds all such instances by a backtrack procedure constructing proofs in a bottom-up left-to-right fashion, using operators from $\Sigma$ in a specific order (the order in which clauses are declared); this last requirement leads to incompleteness, since PROLOG may loop with recursively composable operators, whereas a different order might lead to termination of the procedure. Also, PROLOG may be presented several goals together, and they may share certain variables, but this may be explained by a simple extension of the above proof-synthesis explanation.

We claim that this explanation of PROLOG is more faithful to reality than the usual one with Horn clauses. In particular, our explanation is completely constructive, and we do not have to explain the processes of conjunctive normalization and Skolemization. Furthermore, there is no distinction in $\Phi$ between predicate and function symbols, consistently with most PROLOG iniplementations. 


\subsection{Proof terms with variables, natural deduction.}

The example above demonstraled the difficulty of proofs presented in a Hilbert style. The completely trivial theorem $\forall \boldsymbol{A} \cdot \boldsymbol{A} \rightarrow \boldsymbol{A}$ had a complicated proof using three axioms and two applications of modus poncus. Of course one conld consider adding combinator $I$ as an axiom, but this is only begging the question since other trivial natural theorcms would present similar difficulties. And of course there is no easy way to decide which combinators are well-typed. For instance, Peirce's law:

$$
\text { Peirce }:((A \rightarrow B) \rightarrow A) \rightarrow A
$$

although a propositional tantology easily checkinle by the truth-table method, is not intuitionistically valid.

The natural proof of $A \rightarrow A$ consists in, given a proof $x$ of $A$, returning merely $x$ as a proof of $A$. that is, the natural proof of $A \rightarrow A$ is the (polymorphic) identity algorithm. This unethod of proof usually procecds through the deduction theorem below.

Deduction theorem. Let $\Gamma$ be any set of propositions, $A$ and $B$ be two propositions. We have $\Gamma, A \vdash B$ iff $\Gamma \vdash A \rightarrow B$.

The deduction theorem holds in any reasonable system of logic. It can be proved easily in minimal logic, by induction on the size of proofs. Unfortunately, the deduction theorem is a meta theorem, i.e. a inathernatical theorem of the meta-theory analyzing the proof system, as opposed to the theorems, or well-typed proof terms inside the proof system.

We shall see in section 4 that it is easy to internalize deductions as proof terms with variables, called sequents. This point of view will lead to logic presented in natural deduction style, that is to $\lambda$-calculus formalisms. Before investigating this next level of expressive power, we consider in the next section a particularly important inference system $\Sigma$, that of equational logic.

\section{Rewriting inference and equational logic}

\subsection{The classical presentation}

Equational logic is classically presented as a restriction of first-order logic, where the only predicate symbol is =, and the only non-logical axioms are universal equalities between terms containing free variables. For instance, the theory of groups is classically presented over the functor alphabet

$$
\Phi=\left\{*^{-1}, 1\right\}
$$

by the equations:

$$
\begin{gathered}
\text { Idl }: 1 * x=x \\
\text { Invl: } x^{-1} * x=1 \\
\text { Ass : }(x * y) * z=x *(y * z)
\end{gathered}
$$

and the class of all first order models of these equations is called the varicty of groups. The well known completeness thenrem of Birkhoff states that a universally quantified equation between terms over $\Phi$ is valid in the variety iff it can be deduced from the axioms using the rules of substitution and of replacement of equal for equal. 


\subsection{The proof-theoretic formalization}

Here we ignore the alsstract notion of nodel and concentrate on the rules of inference. We assume given a functor alphabet $\Phi$ given with arily function $\alpha$, in which we distinguish an atom $\rightarrow$ given with arity 2. The substitution inference rule disappears, since it is implicit from the polymorphism of other rules. The replarement of equals for equals is decomposed into elementary steps of term replacement rulcs:

$$
\begin{gathered}
I d_{A}: A \rightarrow A \\
;: \frac{A \rightarrow B \rightarrow C}{A \rightarrow C}
\end{gathered}
$$

Reflexivity

Transitivity

which specify that the rewriting arrow $\rightarrow$ is a quasi-ordoring. Now we must state that $\rightarrow$ is compatible with the rest of the $\Phi$-structure. That is, for every functor $F$ in $\Phi-\{\rightarrow\}$ of arity $n$ and for every $i \leq n$ we take a congruence rule:

$$
\text { Funct } F_{F, i}: \frac{A \rightarrow B}{F\left(A_{1}, \ldots, A_{i-1}, A, A_{i+1}, \ldots, A_{n}\right) \rightarrow F\left(A_{1}, \ldots, A_{i-1}, B, A_{i+1}, \ldots, A_{n}\right)} \quad \text { Congruence }
$$

If we add the rule of symmetry we get the theory of equality, where we usually use symbol = instead of $\rightarrow$ :

$$
O p: \frac{A=B}{B=A}
$$

Symmetry

'The non-logical axioms of the variety are then added as so many constants. For instance, over groups, we obtiin a proof of proposition $y=x^{-1} *(x * y)$ by the term

$$
O p\left(F \text { unct }_{*, 1}(\operatorname{Invl}) ; I d l\right) ; \text { Ass : } y=x^{-1} *(x * y)
$$

Exercise: Show a proof of $x * 1=x$ using the inference system $\Sigma$ above.

The conclusion we may draw from the example above is that, beyond its apparent simplicity, equational reasoning may indeed be quite complicated. The rule of symmetry is specially hard to use since it expresses a commutativity of $\rightarrow$, harder to visualize than the easior monoid structure implicit from the rules $I d$ and ";". It is then natural to ask:

1) Can we climinate $\mathrm{Op}$

2) More generally, can we normalize equational proofs?

\subsection{The categorical viewpoint}

This viewpoint gives a prominent role to the monoïd structure of the quasi-ordering $\rightarrow$. Simplifying the presentation, we may present a category as prescnted by a set of objects $O b j$, which we shall here confuse with the set of (closed) terms over some functor alphabet $\Phi$, and by a set of arrows (or morphisins) which we shall here confuse with the set of (closed) proofs generated from some inference system $\Sigma$ containing initially the two rules:

$$
\begin{gathered}
I d_{A}: A \rightarrow A \\
;: \frac{A \rightarrow B B \rightarrow C}{A \rightarrow C}
\end{gathered}
$$

Identity

Composition

Whenever $\int: A \rightarrow B$, we say that arrow $\int$ has domain $A$ and colominin $B$. Furthermore, it is specified that the proofs are quotiented by a congruence $=$ verifying:

$$
\bar{I} d i l: \bar{l} d ; \hat{f}=f
$$




$$
\begin{gathered}
I d r: \int ; I d=f \\
\text { Ass: }(f ; g) ; h=f ;(g ; h)
\end{gathered}
$$

So we see that a catcyory is a structure obtained as a hybrid of quasi-ordering and of monoïl, to which it reduces in the two degencrate cases (i.e. $|f: A \rightarrow B| \leq 1$ and $|O b j|=: 1$ ). Note that we have given the saune name to axion Inl as for the axiomatization of groups alove, althongh here the operator ";" is a $\Sigma$-operator, and not just a $\$$-operator like "k". Fowever the unification theorem allows us to make implicit the type of variable $f$ above, and the overloading of "Idl" may bo seen as a reflection principle.

If $\mathbf{A}$ and $\mathbf{B}$ are two categories, a functor $F$ from $\mathbf{A}$ to $\mathbf{B}$ associates to every object $\mathbf{A}$ of $\mathbf{A}$ an object. $F(A)$ of $\mathrm{B}$, and to every arrow $f: A \rightarrow B$ an arrow $F(f): F(A) \rightarrow F(B)$ such that the following functorial conditions hold:

$$
\begin{gathered}
F(I d)=I d \\
F(f ; g)=F(f) ; F(g)
\end{gathered}
$$

We see a great analogy between the notion of rewriting inference system and the main catcogrical notions. $\Lambda$ ctually, the categorical viewpoint is richer in that the functors have sorts themselves (i.e., the caterories), and poorer in that they do not yet have arities (i.e. we just have monadic functors so far). In order to build-in arities we shall need products, and a full catcrgorical account of minimal logic is obtained by a further adjunction, namely exponentiation. But we shall defer this explanation until wo develop natural deduction in section 4 . We have given this clementary development of category theory essentially to justify our terminology. The congrucuce rule of terin formation cxplains a functoriality condition on the object part, and the functoriality condition on the arrow part of the functor expresses the congruence property for rewriting. Substitutivity in rewrite rules is expressed by defining them as natural transformations between the functors denoted by the two sides of the rule. That is, a natural transformation $\tau$ betwecn functors $F$ and $G$ (both from category $\mathrm{A}$ to category $\mathrm{B}$ ) is a mapping associating to every object $A$ of $\mathrm{A}$ an arrow $\tau_{A}: F(A) \rightarrow G(A)$ such that

$$
\tau_{A} ; G(j)=F(\hat{f}) ; \tau_{B}
$$

And if we consider equations rather than simply rewrite rules, the symmetry inference rule is intcrpreted as the existence of inverses to arrows. Lquations are thus defined as natural isomorphisms.

Category theory is explained in Mac Lane [04]. The categorical viewpoint for algebra has been developed by Lawvere and others [07]. Its application to proof theory is explained (in a somewhat complicated form) in Szabo [152].

\subsection{Confluence and Termination}

We come back to the problem of eliminating the symmetry rule. Let now $\rightarrow$ be any binary. relation over some set $S, \rightarrow^{*}$ be its reflexive-transitive closure, $\leftrightarrow^{*}$ be its equivalence closurc. We say that $\rightarrow$ verifies the Church-Rosser condition iff

$$
x \leftrightarrow^{*} y \Leftrightarrow \exists z \quad x \rightarrow^{*} z \wedge y \rightarrow^{*} z
$$

It is easy to show that this condition is equivalent to confucnce, i.e.

$$
u \rightarrow^{*}: c \wedge u \rightarrow^{*} y \Leftrightarrow \exists z \quad x \rightarrow^{*} z \wedge y \rightarrow^{*} z
$$

that is, diagrimmatically: 


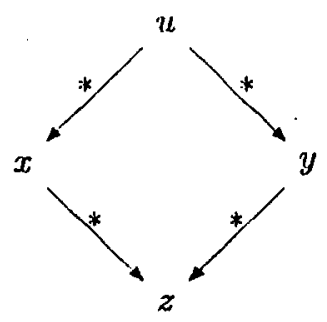

When $\rightarrow$ is a confluent relation, normal forms (i.e. torminal elemonts) are unique whenever they exist, and equality (i.e. $\leftrightarrow^{*}$ ) may be decided by rewriting. That is, deduction may be replaced by computation, and symmetry is eliminated in all but one instance.

For instance, the following set of 10 rewrite rules defines a confluent term rewriting system for group theory:

$$
\begin{gathered}
1 * x \rightarrow x \\
x^{-1} * x \rightarrow 1 \\
(x * y) * z \rightarrow x *(y * z) \\
x * 1 \rightarrow x \\
x * x^{-1} \rightarrow 1 \\
\left(x^{-1}\right)^{-1} \rightarrow x \\
1^{-1} \rightarrow 1 \\
x *\left(x^{-1} * y\right) \rightarrow y \\
x^{-1} *(x * y) \rightarrow y \\
(x * y)^{-1} \rightarrow y^{-1} * x^{-1}
\end{gathered}
$$

The rewrite relation associated with such a term rewriting system $R$ is defined by $M \rightarrow_{n} N$ iff there exists a rule $\alpha \rightarrow \beta$ in $R$ and an occurrence $u$ in $D(M)$ such that $M / u=\sigma(\alpha)$ for some substitution $\sigma$, and $N=M[u \leftarrow \sigma(\beta)]$. It is clear that the group axioms are decided by the system $R$ above. Converscly all rewrite rules in $R$ may be shown to be valid equations in group theory (see the excrcise above). What is less obvious is to decide the confluence of $R$. We shall see in the next section that is is casy to show that it is locally confluent, in the sense that:

$$
u \rightarrow x \wedge u \rightarrow y \Leftrightarrow \exists z \quad x \rightarrow^{*} z \wedge y \rightarrow^{*} z
$$

that is, diagrammatically:

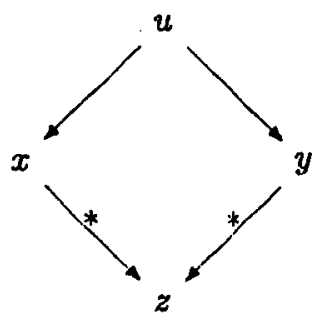


Inwever, local confluenee is not chough to prove confluenes, as shown by the following counterexamples:

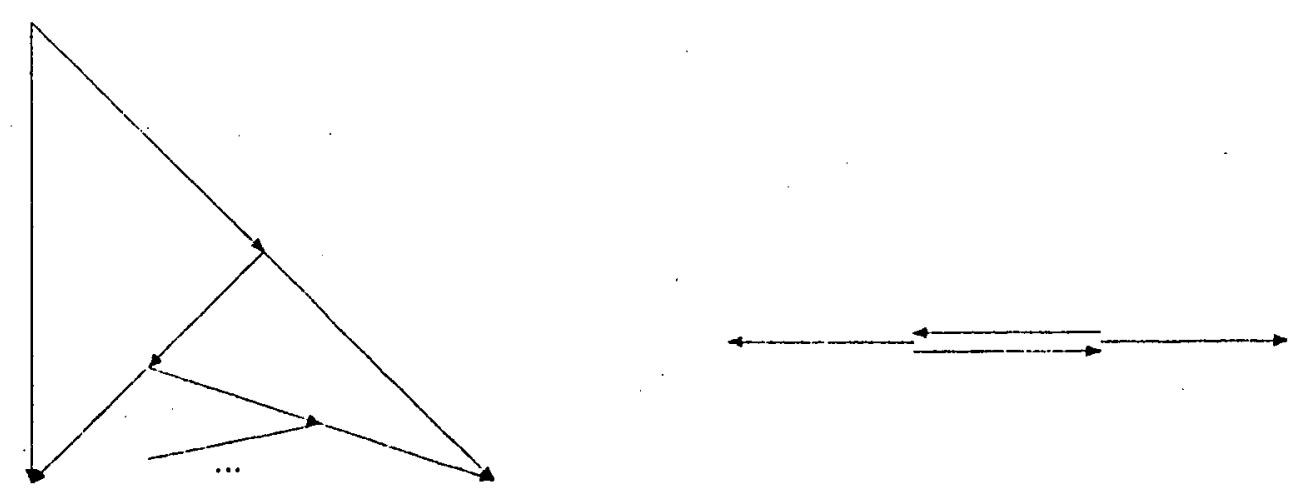

\subsection{The Natherian case: Knuth and Bendix}

The problem encountered with the above counter-examples is that the rewriting relation possessed infinite chains. Let us say that relation $\rightarrow$ is $N$ otherian iff there is no infinite chain $x_{1} \rightarrow x_{2} \rightarrow \ldots$ (Then, its transitive closure $\rightarrow^{+}$is a well-founded ordering). We romark that $\rightarrow$ is Notherian over $S$ iff every non-empty subset of $S$ adnits a minimal element with respect to $\rightarrow^{+}$.

Now let us say that a predicate $P$ over $S$ is $\rightarrow$-hereditary iff

$$
\forall x \in S \quad\left[\forall y \quad x \rightarrow^{+} y \Rightarrow P(y)\right] \Rightarrow P(x) .
$$

Now we may state an important induction principle.

Principle of Notherian Induction: Let $\rightarrow$ be a Nœtherian relation over $S$. Then for every $\rightarrow$-hereditary predicate $P$ we have $\forall x \in S \quad P(x)$.

It is easy to validate this induction principle using the above remark, by considering the set of all $x$ 's such that not $P(x)$. And now we may show that local confluence implies confluence for Notherian relations.

Newman's lemma. A Nothcrian relation is confluent iff it is locally confluent.

Proof: Notherian induction on predidate $P$ defined as:

$$
P(u)=\forall x, y \quad u \rightarrow^{*} x \wedge u \rightarrow^{*} y \Rightarrow \exists z \quad x \rightarrow^{*} z \wedge y \rightarrow^{*} z
$$

We now explain the Knuth-Bendix decision procedure for the confluence of Notherian term rewriting systems. First let us give an algorithm.

Superposition algorithm. Let $\alpha_{1} \rightarrow \beta_{1}$ and $\alpha_{2} \rightarrow \beta_{2}$ bc two rewrite rules in $R$, let $u \in D\left(\alpha_{1}\right)$ and $M=\alpha_{1} / u$ be such that $M$ is a non-variable term unifiable with $\alpha_{2}$. Let $N=\sigma_{1}(M)=\sigma_{2}\left(\alpha_{2}\right)$ be a principal instance, with $V(N) \cap V\left(\alpha_{1}\right)=\emptyset$. We say that the supcrposition of $\alpha_{2} \rightarrow \beta_{2}$ on $\alpha_{1} \rightarrow \beta_{1}$ at $u$ determines the critical pair $\langle P, Q\rangle$, with $P=\sigma_{1}\left(\alpha_{1}\right)\left[u \leftarrow \sigma_{2}\left(\beta_{2}\right)\right]$ and $Q=\sigma_{1}\left(\beta_{1}\right)$.

\section{Examples.}


- $C(I B, x) \rightarrow K(x)$ superpose's on $F(x, G(x, A)) \rightarrow H(x)$ at ' 2 ' to give $P^{\prime}=F^{\prime}(B, K(A))$ and $Q=H(B)$.

- $H(H(x)) \rightarrow K(x)$ superposes on itself at ' 1 ' to give $P=H(K(y))$ and $Q=K(H(y))$.

The Knuth-Bendix theorem. The relation $\rightarrow R$ is locally conflucut iff for cvery critical pair $\left\langle I^{\prime}, Q\right\rangle$ there exists $N$ such that $P^{3} \rightarrow{ }_{R}^{*} N$ and $Q \rightarrow{ }_{R}^{*} N$.

Corollary. If $R$ is a Notherian term rewriting system, its confluence is decidable.

The above theorem may be used to check the locial confluence of the 10 yroup rewrite rules above. Assuming termination, this shows that any group equality many be decided by mere rewriting.

Actually, the method may be extended to systems of rules that fail the test. If for some critical pair $\langle P, Q\rangle$ we reduce $P$ and $Q$ to two distinct irreducible terms $P^{\prime}$ and $Q^{\prime}$, we have generated an interesting lemma $P^{\prime}=Q^{\prime}$, which is an equational consequence of the rules considered as equations. It may be possible to give an orientation to this new equality for forming an extended term rewriting system, while preserving the finite termination property. This is the basis of the Knnth-Bendix cormpletion method, which attempts to complete a term rewriting system to a confluent one. This method may be considered a way of compiling a canonical form algorithm from an equational specification.

We camot describe the method fully here. The main ideas are that unresolved critical pairs are kept as new rewrite rules, and that all rules are kept inter-reduced. The procedure may stop with a canonical system, it may fail because termination is impossible to establish, or it may loop. Whenever it does not fail, it gives a semi-decision procedure for the original equational theory, as explained in Huct [66]. More detailed expositions of the method may be found in $[84,65,71]$.

Pailure may result from some permutative consequence such as commutativity. The method has been extended in various ways in order to consider rewritings modulo such permutative axioms. For instance, Petcrson and Stickel [127] have shown that it was possible to extend the method to complete equational presentations, where one or several functors were assumed to be associative and commutative, using Stickel's associativc-commutative unification algorithm $[151,43]$. This method has been extended by Jonamnaud and Kirchner [73].

Various other extensions of the Knuth-Bendix procedure have been proposed, for handling constructors (free functors) [69] and for solving word problems in finitely presented algebras [90]. The Knuth-Bendix completion procedure and its extensions give a general framework to simplification techniques.

As example of canonical term rewriting system we give distributive lattices. Here $n$ and $U$ are assumed to be associative and commutative. The canonical set consists in the following four rules:

$$
\begin{gathered}
x \cap(x \cup y) \rightarrow x \\
x \cup(y \cap z) \rightarrow(x \cup y) \cap(x \cup z) \\
x \cup x \rightarrow x \\
x \cap x \rightarrow x
\end{gathered}
$$

Exercise. Show that the other distributivity law is a consequence of the above rules.

Finally, we show the canonical system for Boolean algebras. Now the connectives $\wedge$ and $\oplus$ (exclusive or) are assumed to be associative and commutative.

$$
x \wedge 1 \rightarrow x
$$




$$
\begin{gathered}
x \wedge 0 \rightarrow 0 \\
x \wedge x \rightarrow x \\
x \oplus 0 \rightarrow x \\
x \oplus x \rightarrow 0 \\
(x \oplus y) \wedge z \rightarrow(x \wedge z) \oplus(y \wedge z)
\end{gathered}
$$

This canonical set can be used to decide propositional calculus, using the following translations:

$$
\begin{gathered}
\neg x \rightarrow x \oplus 1 \\
x \vee y \rightarrow x \oplus y \oplus(x \wedge y) \\
x \Rightarrow y \rightarrow x \oplus(x \wedge y) \oplus 1
\end{gathered}
$$

The resulting decision method is basically the method of Venn's diagrams, as the following example demonstrates. With three propositional letters $a, b$ and $c$, the proposition

$$
(a \wedge \neg b) \vee(b \wedge \neg c) \vee(c \wedge \neg a)
$$

reduces to its canonical form:

$a \oplus b \oplus c \oplus a \wedge b \oplus b \wedge c \oplus c \wedge a$

which can casily be "seen" as a disjoint union of regions in the following Venn diagram:

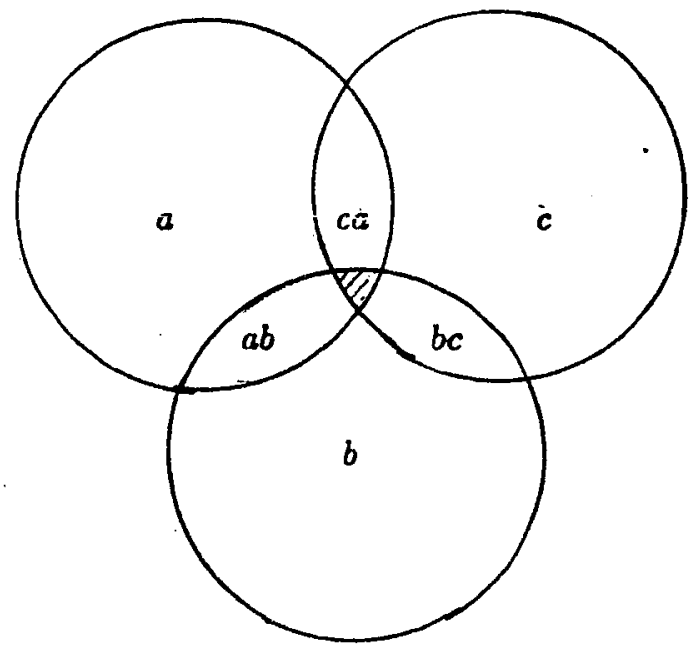

This example also shows that disjunctive normal form is not a canonical form, since the above proposition possesses another d.n.f.

$$
(b \wedge \neg a) \vee(c \wedge \neg b) \vee(a \wedge \neg c)
$$

or, as Quine puts it, a formula may have distinet minimal sots of prime implicants [133]. 


\subsection{Sequential computations}

Wo now consider tem rewriting systons with two constraints:

(a) left linearity: for every $\alpha \rightarrow \beta$ in $R$, every variable of $\alpha$ occurs exactly once

(b) non ambignity: there are no critical pairs

As we shall see, these systems are always confluent, their termination is unnecessary. Functional programming languages, and nuore gencrally operatioual semantics rules can usually be expressed as such systems of rewrite rules [58]. $\Lambda$ s a very simple cxample, consider the system of two rules $D e f_{K}$ and $D e f_{S}$ defining the combinatiors $S$ and $K$.

We shall here define the main notions of computation using rewrite rnlcs. The full theory is given in Iluct-Lévy [70]. We call redox in term $M$ an occurrence $u \in D(M)$ such that $\alpha \leq M / u$ for some left-hand side $\alpha$ of a rule in $R$. We detine the reduction relation $\rightarrow_{R}$ associated with $R$ in the same way as in the preceding section. We shall assume $R$ fixed from now on, and write simply $\rightarrow$ for reduction. Let $M \rightarrow N$ at relex occurrence $u \in D(M)$, using rule $\alpha \rightarrow \beta \in R$. Let now $v$ be any redex in $M$. Wo define the set $v \backslash u$ of residuals of $v$ as a set of redexes in $N$ defined as follows. If $v=u, v \backslash u=\emptyset$. If $v<u$ or $v \mid u$, then $v \backslash u=\{v\}$. Finally, if $v>u$, this means, by non-overlapping, that $v$ is below some variable $x$ of $\alpha$. By lincarity, $x$ has a unique occurrence in $\alpha$, which we shall denote by $x$ as well. That is, $v=u^{-} x^{-} w$ for some $w$. Now let $X$ be the set of occurrences of variable $x$ in $\beta$. We dnfine $v \backslash u=\left\{u-y^{-} w \mid y \in X\right\}$.

Thus redex $v$ may have zero, one or more residuals in $N$. Intuitively, those residuals are the places where one must recluce in $N$ in order to effect the computation step consisting in reducing at redex $v$ in $M$. Actually, on the natural dag implementation all the occurrences of $v \backslash u$ denote the same shared node of the dag representing $N$. Symmetrically the same holds of $u \backslash v$. And as expected we have a local conflucnce diagram, where the single steps $u$ and $v$ confluate using all the steps in $v \backslash u$ (resp. $u \backslash v)$. However, this is not sufficient, since wo do not wint to require $\rightarrow$ to be Notherian. Huwever, it is casy to notice that all the redexes in $v \backslash u$ are mutually disjoint, and that any residual of some redex is always disjoint from any residual of some other disjoint redex. Thus it is natural to extend the reduction relation $\rightarrow$ to parallel reduction of a set of mutually disjoint redexes, a relation we shall write $\#$. If $M \# N$ using set of redexcs $U$, then for every set $V$ of mutually disjoint redexes in $M$, we dofine the residuals of $V$ by $U$ as: $V \backslash U=\{u \in v \backslash u \mid u \in U \wedge v \in V\}$. And now we have a strong confluence property:

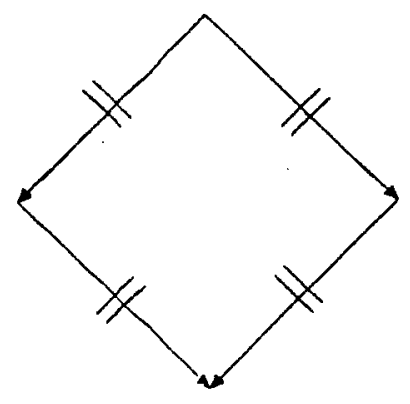

which extcnds easily to multi-steps derivations $A$ and $B$, yielding:

The parallel moves theorem. Let $A$ and $B$ be two co-initial derivationts. Define $A \cup B$ as $A ; B \backslash A$. Then $A \cup B \equiv B \cup A$, in the sense that these two derivations are co-final, and preserve 
residuals.

The categorical viewpoint. The catcgory whose objects are terms, and whose arrows from $M$ to $N$ are parallel derivations, quotinted by the equivalence $\equiv$, admits pushouts.

Corollary. The reduction relation $\rightarrow$ has the Church-Rosser property.

Caution! The lattice structure given by the parallel moves theorem is on derivations, and not on terms. For instance, if we consider the system $R$ consisting solely of the rules $I(x) \rightarrow x$ and $J(x) \rightarrow x$, the following derivations diagram shows that the terms $I(J(K))$ and $J(I(I))$ do not possess a l.u.b.

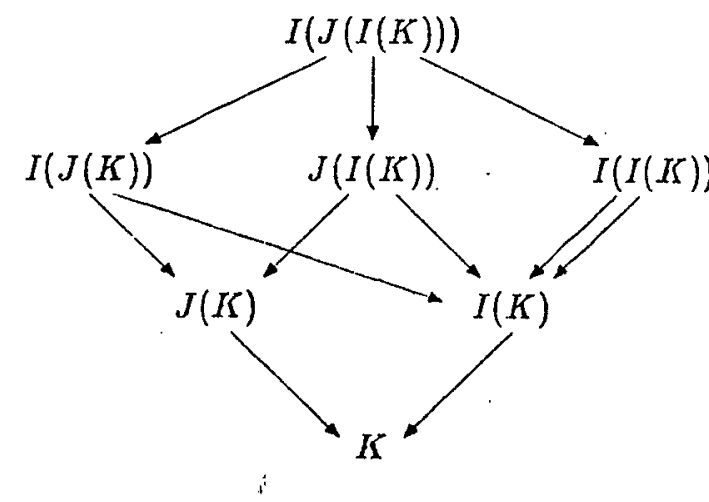

Note that this phenomenon may be traced to the existence of two non-equivalent derivations between $I(I(K))$ and $I(K)$. This shows that the categorical viewpoint is the right one here: we need to talk in terms of arrows, not just relations between termis.

The standardization theorem. It is always possible to compute in an outside-in manner.

We do not have the space here to explain in a rigorous manner what outside-in exactly means. We just remark that this may be more complicated than merely reducing the leftmost-outermost redex, i.e. the redex minimum in the total ordering on occurrences defined by $u<_{L O} v$ iff either $u<v$, or else there exist $u^{\prime} \leq u$ and $v^{\prime} \leq v$ with $u^{\prime}<_{L} v^{\prime}$. For instance, with $R$ consisting of $F(x, D) \rightarrow C, B \rightarrow D$ and $\perp \rightarrow \perp$, the standard derivation going from $F(\perp, B)$ to its canonical form $C$ is:

$$
F(\perp, B) \rightarrow F(\perp, D) \rightarrow C
$$

whereas the leftmost-outermost rule leads to a non-terminating loop.

This theorem suggests it can be used to define a computation rule, usable to drive an interpreter computing "lazily". However, this is not true, since the standard derivation in a derivation class is not simply a function of the starting term. For instance, consilcr Gustave's function, an example due to G.G. Berry; with $R$ consisting of the 3 rules $F(0,1, x) \rightarrow A, F(x, 0,1) \rightarrow A$ and $F(1, x, 0) \rightarrow$ $A$, nothing tells us where to compute next in a term $F\left(M_{1}, M_{2}, M_{3}\right)$. This eximnple is just, as bad as the classical "parallel-or" definition, an obviously non-sequential example ruled out here becunse we do not admit critical pairs.

Thus it is clear that we do not have strong cnough syntiatic restrictions ou the left-hand sides of our rewrite rules to be able to deine sequential computation rules. The key to such restrictions is to adapt the Kahm-Plotkin sequentiality theory [76] to define a notion of "necedel" redex. This 
Jeads to the notion of secpential term rewriting systent. A firther exfinement, stronges secpuentiality, gives a decidable criterion which may be nised to drive efficient interpreters which look for a necded reclex in linear time, using a goncralization to trees of the Kmuth-Mortis-Pratt string-matching alogrithm [85]. This theory is completely cxplained in Inet-Lóvy [70].

In practice, we obtain easy critorions for strong secquentiality in the particular cases of systerns with constructors, and "left" systems such as systcms of combinators definitions.

\section{Natural deduction and $\lambda$-calculus}

\subsection{Proofs with variables: sequents}

We now come back to the general theory of prof structures. We saw earlier that the Hilbert presentation of minimal logic was not very natural, in that the trivial theorem $A \rightarrow A$ necessitated a romplex proof $S K K$. The problem is that in practice one docs not use just proof terms, but derluctions of the form

$$
\Gamma \vdash A
$$

where $\Gamma$ is a sct of (hypothetic) propositions.

Deductions are exactly proof terms with variables. Naming these hypothesis variables and the proof term, we write:

$$
\left\{\ldots\left[x_{i}: A_{i}\right] \ldots \mid i \leq n\right\} \vdash M: A
$$

with $V(M) \subseteq\left\{x_{1}, \ldots, x_{n}\right\}$. Such formulas are called sequents. Since this point of vicw is not very well-known, let us emphasize this constatation:

Sequents represent proof terms with variables.

Note that so far our notion of proof construction has not changed:

$\Gamma \vdash_{\Sigma} M: A$ iff $\vdash_{\Sigma} \cup \Gamma: \Lambda$, i.c. the hypotheses from $\Gamma$ are used as supplementary axioms, in the same way that in the very beginning we have defined $T(\Sigma, V)$ as $T(\Sigma \cup V)$.

\subsection{The deduction theorem}

This theorem, fundamental for doing proofs in practice, gives an equivalence between proof terms with variables and functional proof terms:

$$
\Gamma \cup\{A\} \vdash B \Leftrightarrow \Gamma \vdash A \rightarrow B
$$

That is, in our notations:

a) $\Gamma \vdash M: A \rightarrow B \Rightarrow \Gamma \cup\{x: A\} \vdash(M x): B$

This direction is immediate, using App, i.e. Modus Ponens.

b) $\Gamma \cup\{x: A\} \vdash M: B \Rightarrow \Gamma \vdash[x] M: A \rightarrow B$

where the term $[x] M$ is given by the following algorithm.

Schönfinkel's abstraction algoritlım:

$$
\begin{array}{ll}
{[x] x=I \quad} & (=S K K) \\
{[x] y=K y \quad(y \neq x)} & \\
{[x](M N)=S} & {[x] M[x] N}
\end{array}
$$


Note that this algorithm motivates the choice of combinalors $S$ and $K$ (and optionally $I$ ). Again we stress a basic olservation:

Schönfinkel's algorithm is the essence of the proof of the deduction theorem.

Now let us consider the rewriting system $R$ defined by the rules $D e f_{K}$ and $D e \int_{S}$, optionally supplomented by:

$$
\operatorname{Def}_{I}: I x=x
$$

and let us write $\triangleright$ for the corresponding reduction relation.

Fact. $\quad([x] M N) \quad D^{*} M[x \leftarrow N]$.

We leave the proof of this very important property to the reader. The important point is that the abstraction operation, together with the application operator and the reduction $\triangleright$, define a substilution machinery. We shall now use this idlea more generally, in order to internalize the deduction theorem in a basic calculus of functionality. That is, we forget the specific combinators $S$ and $K$, in favor of abstraction seen now as a new term constructor.

\section{$4.3 \lambda$ calculus.}

Here we give up $\Sigma$-terms in general, in favor of $\lambda$-terms constructed by 3 elcmentary operations:

This last case is usually written $\lambda x \cdot M$, whence the name $\lambda$-notation. The $\lambda$-notation is first a non-ambiguous notation for expressions denoting functions. For instance, the function of two arguments which computes sin of its first argument and adds it to cos of its second is written

$$
[x][y] \sin (x)+\cos (y)
$$

The variables $x$ and $y$ are bound variables, that is they are dummies and their name does not matter, as long as there are no clashes. This defines a congruence of renaming of bound variables usually called $\alpha$-conversion. Another method is to adopt de Bruijn's indexes, where variable names disappear in favor of positive natural numbers [15]. We define recursively the sets $\lambda_{n}$ of $\lambda$-expressions valid in a context of length $n \geq 0$ as follows:

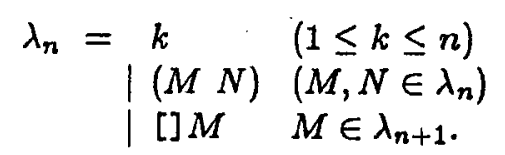

Thus integer $n$ refers to the variable bound by the $n$-th abstraction above it. For instance,

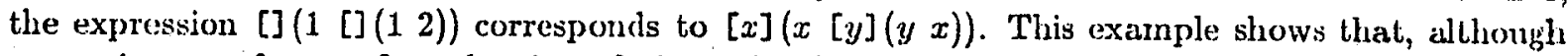
more rigorous from a formal point of view, the de Bruijn naming scheme is not fit for human understanding, and we shall now come back to the more usual concrete notation with variable names. 
The fact observed above is now edicted as in computation rule, usually called $\beta$-returtion. Jet $\triangleright$ be the sunallest relation on $\lambda$-expressions compatible with application and abstiaction and such that:

$$
([x] M N) \triangleright M[x \leftarrow N] .
$$

We call $\lambda$-calculus the $\lambda$-notation cquipped with the $\beta$-reduction computation rule $\triangleright$. $\lambda$-calculus is the basic calculus of substitution, and $\beta$-reduction is the basic computation mechanism of functional programming languages. Here is an example of computation:

$$
\begin{gathered}
([x][y](x(y x))[u](u u)[v][w] v) \\
\triangleright([y]([u](u u)(y[u](u u)))[v][w] v) \\
\triangleright^{2}([u](u u)[w][u](u u)) \\
\triangleright([w][u](u u)[w][u](u u)) \triangleright[u](u u)
\end{gathered}
$$

We briefly sketch here the syntactic properties of $\lambda$-calculus. Similarly to the theory reveloped above, the notion of residual can be defined. However, the residuals of a redex may not always be disjoint, and thus the theory of derivations is more complex. However the parallel moves lemma still holds, and thus the Church-Rosser property is also true. Finally, the standardization theorem holds, and here it means that it is possible to compute in a leftmost-outcrmost fashion. These results, and more details, in particular the precise conditions under which $\beta$-reduction simnlates combinatory logic calculus, are precisely stated in Barendrogt [4].

We finally remark that $\lambda$-calculus computations may not always terminate. For instance, with $\Delta=[u](u u)$ and $\perp=(\Delta \Delta)$, we get $\perp \triangleright \perp \triangleright \ldots$ A more interesting example is given by

$$
Y=[f]([u](f(u u))[u](f(u u)))
$$

since $(Y f) D^{*}(f(Y f))$ shows that $Y$ defines a general fixpoint operator. This shows that (full) $\lambda$-calculus is inconsistent with logic. What could $(f i x \neg)$ mean? As usual with such paradoxical situations, it is necessary to introduce types in order to stratify the definable notions in a logically meaningful way. Thus, the basic inconsistency of Church's $\lambda$-calculus, shown by Rossece, led to Church's theory of types [22]. On the other hand, $\lambda$-calculus as a pure computation mechanism is perfectly meaningful, and Strichey prompted Scott to develop the theory of reflexive domains as a model theory for full $\lambda$-calculus. But let us first investigate the typed universe.

\subsection{Gentzen's system N of natural deduction}

The idea of $\lambda$-notation proofs underlies Gentzen's natural deduction inference rules [48], where App is called $\rightarrow$-clim and $A b s$ is called $\rightarrow$-intro. The role of variables is taken by the base secuuents:

$$
\text { Axiom }_{A}: A \vdash A
$$

together with the structural thinning rule:

$$
\text { Thinning : } \frac{\Gamma \vdash B}{\Gamma \cup\{A\} \vdash B}
$$

which expresses that a proof may not use all of the bypotheses. Gent\%en's remaining rules give types to proofs according to propositions built as functor ter:ns, each functor corresponding to a proposition al connective. The mian idea of his system is that inference rules should not be arbitrary, 
but should follow the functor structure, in explaining in a uniform fawhion how to introduce a functor, and how to climinatio it. For instance, mininal logic is obtained with $\Phi=\{\rightarrow\}$, and the rules of $\rightarrow$-..intro and $\rightarrow-$ elim, that is:

$$
\begin{gathered}
A b s: \frac{\Gamma \cup\{A\} \vdash B}{\Gamma \vdash A \rightarrow B} \\
A p p: \frac{\Gamma \vdash A \rightarrow B \Delta \vdash A}{\Gamma \cup \Delta \vdash B}
\end{gathered}
$$

Now, the $\beta$-rcduction of $\lambda$-calculus corresponds to cut-elimination, i.e. to proof-simplification. Reducing a redex corresponds to eliminating a detour in the denonstration, using an intermediate lomma. But now we have termination of this normalization process, that is the relation $\rightarrow$ is Noxtherian on valid proofs. This result is usually called strong normalization in proof theory. A full account of this theory is given in Stenlund [150].

Minimal logic can then be extended by adding more functors and corresponding inference rules. For instance, conjunction $\wedge$ is taken into account by the intro rule:

$$
\text { Pair : } \frac{\Gamma \vdash A \Delta \vdash B}{\Gamma \cup \Delta \vdash A \wedge B}
$$

which, from the types point of view, may be considered as product formation, and by the two clim rules:

$$
\begin{aligned}
\text { Fst } & : \frac{\Gamma \vdash A \wedge B}{\Gamma \vdash A} \\
\text { Snd }: & \frac{\Gamma \vdash A \wedge B}{\Gamma \vdash B}
\end{aligned}
$$

corresponding to the two projection functions. This corresponds to building-in a $\lambda$-calculus with pairing. Generalizing the notion of redex (cut) to the ccinfiuration of a connective intro, immediately followed by elim of the same connective, we get new computation rules:

$$
\begin{aligned}
& F s t(\operatorname{Pair}(x, y)) \triangleright x \\
& \operatorname{Snd}(\operatorname{Pair}(x, y)) \quad \triangleright y
\end{aligned}
$$

and the Notherian property of $\triangleright$ still holds. We shall not develop further Gentzen's system. We just remark:

(a) More connectives, such as disjunction, can be added in a similar fashion. It is also possible to give rules for quantifiers, although we prefer to differ this topic until we consider dependent bindings.

(b) Gentzen originally considered natural deduction systems for meta-mathematical reasons, namely to prove their consistency. He considered another presentation of sequent inference rules, the L system, which possesses the subformula property (i.e. the result type of every operator is formed of subterms of the argument types), and is thus trivially consistent. Strong normalization in this context was the essential technical tool to establish the equivalence of the $\mathrm{L}$ and the $\mathrm{N}$ systems. Of course, according to Gödel's theorem, this does not estallish absolute consistency of the logic, but relativi\%es it to a carefully identified troublesome point, the proof of termination of some reduction relation. This has the additional advantage to provide a hierarchy of strength of inference systems, classificd according to the ordinal necessary to consider for the termination proof. 
(c) All this development concerns so called intuitionistic loyic, where operators (inference males) are deterministic. It is possible to gencralize the inference systems to classical logic, using it gencralizes notion of sepuent $\Gamma \vdash \Delta$, where the right part $\Delta$ is also a set of propesitions. It is possible to explain the composition of such non-delerministic operators, which leauls to Gentzen's systems NK aud LK (Klassical logic!). Remark that the analogue of the unification theorem above: gives then precisely Robinson's resolution principle for general clanses [140].

(d) The categorical viewpoint fits niccly these devclopments. This point of vicw is completely developed in Szabo [152]. The specially important connections between $\lambda$-calculus, natural deduction proufs and cartesian closed categories are investigated in $[98,121,87,143,35,68]$. Further readings on natural deduction proof theory are Prawitz [130] and Dummett [41]. The connection with recursion theory is developed in Kleene [82] and an algcbraic treatment of these matters is given in Rasiowa-Sikorski [134].

\subsection{Programming languages, recursion}

The design of programming languages such as ALGOL 60 was greatly influenced by $\lambda$-calculus. In 1966 Petcr Landin wrote a landmark article setting the stage for coherent design of jowcrful functional languages in the $\lambda$-calculus tradition [80]. The core language of his proposal, ISWIM (If you see what I mean!) meant $\lambda$-calculus, with syntactically sugared versions of the $\beta$-redex ([x]MN), namely let $x=N$ in $M$ and $M$ where $x=N$. respectively. His language followod the static binding rules of $\lambda$-calculus. For instance, after the declarations:

$$
\begin{aligned}
& \text { let } f x=x+y \text { where } y=1 \text {; } \\
& \text { let } y=2 \text {; }
\end{aligned}
$$

the evaluation (reduction) of expression $(f 1)$ Ieads to value 2 , as expected. Note that in contrast languages such as LISP [107], although bearing some similarity with the $\lambda$-notation, implement rather dynamic binding, which would result in the example above in the incorrect result 3 . This discrepancy has led to heated debates which we want to avoid herc, but we remark that static binding is generally considered safer and leads to more efficient implementations where compilation is consistent with interpretation. However, ISWIM is not completely faithful to $\lambda$-calculus in one respect: its implementation does not, follow the outside-in normal order of evaluation corresponding to the standardization theorem. Instead it follows the inside-out applicative order of evaluation demanding the arguments to be evaluated before a procedure is called. In the A.LGOL terminology, ISWIM follows call by value instead of call by name.

The development of natural deduction as typed $\lambda$-calculus fits the design of an ISWIM-based language with a type discipline. We shall call this language ML, which stands for "meta-language", in the spirit of LCF's ML $[54,53]$. For instance, we get a core $\mathrm{ML}_{0}$ by considering minimal logic, with $\rightarrow$ intcrpreted as functionality, and further constant functors added for basic types such as triv, bool, int and string.

Adding products we get a language $\mathrm{ML}_{1}$ where types refiect an intuitionistic predicate calculus with $\rightarrow$ and $\wedge$. We nnay define functions on a pattern argument formed by pairing, such as:

$$
\text { let } f \operatorname{st}(x, y)=x
$$

and the categorical analogue are the so-called carlesian closed catcgories (CCCs). Adding sums lead to Bi-CCC's with co-product. The corresponding $\mathrm{ML}_{2}$ primitives are inl, inr, outl, outr and isl, with obvious meaning. So far all computations terminate, since the corresponding reduction relations are Notherian. 
However such in poggamming language is too weak for practical use, since recursion is missing. Adking recursion operators may be done in a stratilied manner, ats presented in Gödel's system $\mathrm{T}$ [51], or in a completely general way in $\mathrm{ML}_{3}$, where we allow a "letece" construct permitting arbitrary recursive definitions, such as:

$$
\text { letrec } \int \text { act } n=\text { if } n=0 \text { then } 1 \text { else } n *\left(\int a c t(n-1)\right)
$$

But then we loose the termination of computations, since it is possible to write un-founded definitions such as

$$
\text { letrec absurd } x=\text { absurd } x \text {. }
$$

Furthermore, because ML follows the applicative order of evaluation we may get looping computations in cases where a $\lambda$-calculus normal form exists, such as for

$$
\text { let } f x=0 \text { in } f(a b s u r d x) \text {. }
$$

\subsection{Polymorphism}

We have polymorphic operators (inference rules) at the neta level. It secms a good idea to push polymorphism to the object level, for functions defined by the user as $\lambda$-expressions. To this end, we introduce bindings for type variables. This idea of type quantification corresponds to allowing proposition quantifiers in our propositional logic. First we allow a universal quantifier in prenex position. That is, with $T_{0}=T(\Phi, V)$, we now introduce type schemas in $T_{1}=T_{0} \cup \forall \alpha T_{1}, \alpha \in V$. A (type) term in $T_{1}$ has thus both free and bound variables, and we write $F V(M)$ and $B V(M)$ for the sets of free (respectively bound) variables.

We now define gencric instanciation.

Let $\tau=\forall \alpha_{1} \ldots \alpha_{m} \cdot \tau_{0} \in T_{1}$ and $\tau^{\prime}=\forall \beta_{1} \ldots \beta_{n} \cdot \tau_{0}^{\prime} \in T_{1}$. We define $\tau^{\prime} \geq_{G} \tau$ iff $\tau_{0}^{\prime}=\sigma\left(\tau_{0}\right)$ with $D(\sigma) \subseteq\left\{\alpha_{1}, \ldots, \alpha_{m}\right\}$ and $\beta_{1} \notin F V(\tau) \quad(1 \leq i \leq n)$. Remark that $\geq$ acts on $F V$ whereas $\geq G$ acts on $B V$. Also note

$$
\tau^{\prime} \geq_{G} \tau \Rightarrow \sigma\left(\tau^{\prime}\right) \geq_{G} \sigma(\tau)
$$

We now present the Damas-Milner inference system for polymorphic $\lambda$-calculus [39]. In what follows, a sequent hypothesis $A$ is assumed to be a list of specifications $x_{i}: r_{i}$, with $r_{i} \in T_{1}$, and we write $F V(A)=\bigcup_{i} F V\left(\tau_{i}\right)$.

$$
\begin{gathered}
T A U T: A \vdash x: \alpha \quad(x: \alpha \in A) \\
\left.I N S T: \frac{A \vdash M: \alpha}{A \vdash M: \beta} \quad \alpha \leq G \beta\right) \\
G E N: \frac{A \vdash M: \tau}{A \vdash M: \forall \alpha \cdot \tau \quad(\alpha \notin F V(A)} \\
A P P: \frac{A \vdash M: \tau^{\prime} \rightarrow \tau A \vdash N \tau^{\prime}}{A \vdash(M N): \tau} \\
A B S: \frac{A \cup\left\{x: \tau^{\prime}\right\} \vdash M: \tau}{A \vdash[x] M: \tau^{\prime} \rightarrow \tau} \\
L E T: \frac{A \vdash M: \tau^{\prime} A \cup\left\{x: \tau^{\prime}\right\} \vdash N: \tau}{A \vdash l e t: x=M i n N: \tau}
\end{gathered}
$$


For instance, it is an easy cxercise to show that

$$
\text { -let } i=[x] x \text { in }(i \quad i): \alpha \rightarrow \alpha \text {. }
$$

The above system may be extomded withont difficulty by other functors such as product, and by other ML contructions such as letrec. Actually every ML compiler contanins a typechecker implementing implicitly the above inference system. For instance, with the wuary functor list and the following ML primitives: [] : (list $\alpha$ ), cons : $\alpha \times($ list $\alpha)$ (written infix as a lot), hd: $($ list $\alpha) \rightarrow \alpha$ and $l l:($ list $\alpha) \rightarrow($ list $\alpha)$, we may define recursively the map functional as:

$$
\text { letrec map } f l=\text { if } l=[] \text { then }[] \text { else }(f(h d l)) \cdot \operatorname{map} f(t l l)
$$

and we get as its type:

$$
\text { ․ } \operatorname{map}:(\alpha \rightarrow \beta) \rightarrow(\text { list } \alpha) \rightarrow(\text { list } \beta) .
$$

Of course the ML compiler does not implement directly the inference system ahove, which is non-deterministic because of rules $I N S T$ and $G E N$. It uses unification instcad, and thus computes deterministically a principal type, which is minimum with respect to $\leq_{G}$ :

Milner's Theorem. Every typable expression of the polynorphic $\lambda$-caleulus possesses a principal type, minimum with respect to generic instanciation.

We obtain $M_{4}$ by restricting $\mathrm{ML}_{3}$ to the type system above. $\mathrm{ML}_{4}$ is a strongly typed programming language, where type inference is possible because of the above theorem: the user need not write type specifications. The compiler of the language does more than typechecking, since it actually performs a proof synthesis. Types disappear at run time, but because of the type analysis no dynamic checks are needed to inforce the consistency of data operations, and this allows fast exccution of ML programs. ML is a generic name for languages of the ML fanily. For instance, by adding to $\mathrm{ML}_{4}$ exceptions, abstract data types (permitting in particular user-defined functors) and references, one gets approximately the meta-language of the LCF proof assistant [54]. By adding record type declarations (i.e. labeled sums and products) one gets L. Cardelli's ML [20]. By adcling constructor types, pattern-matching and concrete syntax, we get the Ije ML langunge under development in the Formel project [33]. A more complete language, including modulcs, is under design as Standard ML [112]. It is to be hoped than less than 700 itcrations of the language design will be necessary before the ultimate Standard ML is agreed upon [89]! Current research topics on the design of ML -like languages are incorporation of object-oriented features allowing subtypes, remanent data structures and bitmap operations [21]: and "lazy evaluation" permitting streams and ZF expressions $[162,118]$.

Note on the relationship between ML and $\lambda$-calculus. First, ML uses so-called call by value implementation of procedure call, corresponding to innermost reduction, as opposed to the ontermost regime of the standard reduction. Lazy evaluation permits standard reductions, but closures (i.e. objects of a functional type $\alpha \rightarrow \beta$ ) are not evaluated. Finally, types in ML serve for insuring the integrity of data operations, but still allow infinite computations by non-terminating recursions.

\subsection{The limits of ML 's polymorphism}

Consider the following ML definition:

$$
\begin{gathered}
\text { letrec power } n f u=\text { if } n=0 \text { then } u \\
\text { else } f(\text { power }(n-1) f u)
\end{gathered}
$$


of type nat $\rightarrow(\alpha+\alpha) \rightarrow(\alpha \rightarrow \alpha)$. This function, which associates to natural n the polymorphic

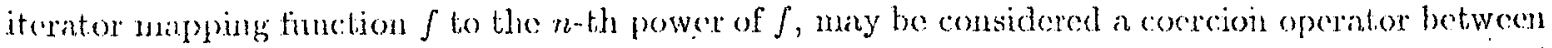
ML 's internal naturals and Church's representation of naturals in prure $\lambda$-calculus [20]. Let us recall bricfly this representation. Integor 0 is represented as the projection torm $[f][u] u$. Integer 1 is $\left[\int\right][u]\left(\int u\right)$. More generally, $n$ is represented as the functional $\bar{n}$ iterating a function $\int$ to its $n$-th power:

$$
\bar{n}=\left[\int\right][u]\left(\int\left(f \ldots\left(\int u\right) \ldots\right)\right)
$$

and the arithmetic: opcrators may be coded respectively as:

$$
\begin{gathered}
n+m=\left[\int\right][u]\left(n f\left(m \int u\right)\right) \\
n \times m=\left[\int\right](n(m f)) \\
n^{m}=(m n)
\end{gathered}
$$

For instance, with $\overline{2}=\left[\int\right][u](f(f u))$, we check that $\overline{2} \times \overline{2}$ converts to its normal form $\overline{4}$.

We would like to consider a type

$$
N A T=\forall \alpha \cdot(\alpha \rightarrow \alpha) \rightarrow(\alpha \rightarrow \alpha)
$$

and be able to type the operations above as functions of type $N A T \rightarrow N A T \rightarrow N A T$. However the notion of polymorphism found in ML does not support such a type, it allows only the weaker

$$
\forall \alpha \cdot((\alpha \rightarrow \alpha) \rightarrow(\alpha \rightarrow \alpha)) \rightarrow((\alpha \rightarrow \alpha) \rightarrow(\alpha \rightarrow \alpha)) \rightarrow((\alpha \rightarrow \alpha) \rightarrow(\alpha \rightarrow \alpha))
$$

which is inadequate, since it forces the same generic instanciation of $N A T$ in the two arguments.

Warning. These preliminary notes are very sketchy from now on. A future version will cover the topics below in greater depth.

\subsection{Girard's second order $\lambda$-calculus.}

The example above suggests using the universal type quantifier inside type formulas. We thus consider a functor alphabet based on one binary $\rightarrow$ constructor and one quantifier $\forall$. We shall now consider a $\lambda$-calculus with such types, which we shall call second-order $\lambda$-calculus, owing to the fact that the type language is now a second-order propositional logic, with propositional variables explicitly quantified. Such a calculus was proposed by J.Y. Girard [40,50], and independently discovered by J. Reynolds [136].

Girard proved the main properties of the calculus:

Girard's theorem. Second-order $\lambda$-calculus admits strong normalization.

Corollary. Second-order natural deduction is consistent.

Girard used this last result to show the consistency of analysis.

Second-order $\lambda$-calculus is a very powerful language. Most usual data structures may be represented as types. Furthormore, it captures a large class of total recursive functions (precisely, all the functions prouvalbly total in sceond-order arithmetic). It may seriously be considered as a candidate for the foundations of powerful programming languages, where recursion is replaced hy iteration. But the price we pay by extending polymorphism in this drastic fashion is that the notion of principal type is lost. Type synthesis is possible only in easy cascs, and thus in general the programmer has to specify the types of its data.

Further discussions on the second-order $\lambda$-calculus may be found in $[108,46,01,7]$. 


\section{Dependent types}

\subsection{Quantification}

So far we have dealt only with types as propessitions of some (intuitionistic) propesitional logic. We shall now consider stronger logics, where it is possible to have statements depending upon variables that are $\lambda$-bound. We shall contimuc our identification of propositions and types, and thus consider a first-order statcoment such as $\forall x \in E \cdot P(x)$ as a product-forming type $\prod_{x \in E} P(x)$.

We slall call such types dependent, in that it is now possible to declare a variable of a type which depends on the binding of some previously bond variable. Let us first of all remark that such types are absolutely necessary for practical programming purposes. For instance, a matrix manipulation procedure should have a clectaration profix of the type:

$$
[n: n a t][\text { matrix : } \operatorname{array}(n)]
$$

where the sccond type depends on the dimension parameter. PASCAL programmers know that the lack of such declarations in the linguage is a serious hindrance.

Wc shall not develop first-order notions here, and shall rather jump directly to calculi based on higher-order logic.

\subsection{Martin-Löf's Intuitionistic Theory of Types}

P. Martin-Löf has been developing for the last 10 years a higher-order intuitionist logic based on a theory of types, allowing dependent sums and products $[104,105,106]$. His theory is not explicitly based on $\lambda$-calculus, but it is formulated in the spirit of natural deduction, with introduction and elimination rules for the various type constructors. Consistency is inferred from semantic considerations, with a model theory giving an analysis of the normal forms of clements of a type, and of the equality predicate for each type.

Martin-Lüf's system has been advocated as a good candidate for the description and validation of computer programs, and is an active topic of research by the Gotteborg Programuning Methodology group [117,110,120]. A particularly ambitious implementation of Martin-Löf's system and extensions is under way at Cornell University, under the direction of $R$. Constable $[25,26,132]$.

\section{3 de Bruijn's AUTOMATH languages}

The mathematical language AUTOMA'TH has been developed and implemented by the Eindhoven group, under the direction of prof. N.G. de Bruijn $[14,16,18]$. AUTOMATH is a $\lambda$-calculus with types that are themselves $\lambda$-expressions. It is based on the ratural idea that $\lambda$-binding and universal instanciation are similar substitution operations. Thus in AUTOMATH there is only one binding operation, used both for parameter abstraction and product instanciation. The meta-theory of the various langiages of the AUTOMATH family are investigated in $[113,38,75]$. The most notable success of the AUTOMATH effort has been the translation and mechinical valiclation of Landau's Grundlagen [74]:

\subsection{A Calculus of Constructions.}

AUTOMATH established the correct linguistic foundations for higher-order natural deduction. Unfortunately, it did not allow Girard's second-order types, and probably for this reason was never considered under the programming language aspect. Th. Coquand showed that a slight extension of 
the notation allowed the incorporation of Girard's typess to AUTOMATH in a natual mamner [27]. Conpand showed by a strong nomalization theorem that the formalism is consistent. Experinents with an inplementation of the calculus showed that it is wcll adauted to expressing naturally and conciscly mathenatical proofs and computer algorithms [29]. Vartations on this calculns are under devclopmont $[30,31]$.

\section{Conclusion}

We have presented in these notes a uniform account of logic and computation theory, based on proof theory notions, and most importantly on the Curry-Howard correspondance between propositions and types $[37,50]$.

These notes are based on a course given at the Advanced School of Artificial Intelligence, Vigneu, France, in July 1985. An extended version is in preparation.

\section{References}

[1] A. Aho, J. Hopcroft, J. Ullman. "The Design and Analysis of Computer Algorithms." Addison-Wesley (1974).

[2] P. B. Andrẹws. "Resolution in Type Theory." Journal of Symbolic Logic 36,3 (1971): 414432.

[3] P. B. Andrews, D. A. Miller, E. L. Cohen, I. Pfenning. "Automating higher-order logic." Dept of Math, University Carnegie-Mellon, (Jan. 1983).

[4] H. Barendregt. "The Lambda-Calculus: Its Syntax and Semantics." North-Holland (1980).

[5] E. Bishop. "Foundations of Constructive Analysis." McGraw-Hill, New-York (1967).

[6] F. Bishop. "Mathernatics as a numerical language." Intuitionism and Proof Theory, Eds. J. Myhill, A.Kino and R.E.Vesley, North-Holland, Amsterdam, (1970) 53-71.

[7] C. Böhm, A. Berarducci. "Automatic Synthesis of Typed Lambda-Programs on Term Algebras." Unpublished manuscript, (June 1984).

[8] R.S. Boyer, J Moore. "The sharing of structure in theorem proving programs." Machine Intelligence 7 (1972) Edinburgh U. Press, 101-116.

[9] R. Boyer, J Moore. "A Lemma Driven Automatic Theorem Prover for Recursive Function Theory." 5th International Joint Conference on Artificial Intelligence, (1977) 511-519.

[10] R. Boyer, J Moore. "A Computational Logic." Academic Press (1979).

[11] R. Boyer, J Moore. "A mechanical proof of the nnsolvability of the halting problem." Report ICSCA-CMP-28, Institute for Computing Science, Univcrsity of Texas at Austin (July 1982).

[12] R. Boyer, J Moore. "Proof Checking the RSA Public Key Encryption Algorithm." Repurt ICSCA-CMP-33, Institute for Computing Science, University of Texas at, Austin (Sept. 1082).

[13] R. Boyer, J Moore. "Proof cliecking theorem proving and program verification." Report ICSCA-CMP-35, Institute for Computing Science, University of Texas at Austin (Jan. 1983). 
[14] N.G. de Brnijn. "The mathematical lang"atge AUTOMATH, its uxage and soune of its ext.ensions." Symposium on Automatic Demonstration, IRIA, Versailles, 1968. Printed as Springer-Verlaty Lecture Notes in Mathematics 125, (1970) 29-61.

[15] N.G. de Bruijn. "Lambda-Calculus Notation with Namcless Dummies, a Tool for Automatic Formula Manipulation, with Application to the Church-Rosser Theorem." Indag. Math. 34,5 $(1972), 381 \cdots 392$.

[16] N.G. de Bruijn. "Automath a language for mathematics." Les Presses de l'Université de Montréal, (1973).

[17] N.G. de Bruijn. "Some extensions of Automath: the AUT-4 fanily." Internal Automath memo M10) (Jin. 1974).

[18] N.G. de 13ruijn. "A survey of the project Automath." (1980) in to H. B. Curry: Essays on Combinatory Logic, Lambda Calculus and Formalism, Eds Seldin J. P. and Hindley J. IR., Academic Press (1980).

[19] M. Bruynooghe. "The Mcmory Management of PROLOG implementations." Logic Programming Workshop. Ed. Tarnlund S.A (July 1980).

[20] L. Cardelli. "ML under UNIX." Bell Laboratories, Murray Hill, New Jersey (1982).

[21] L. Cardelli. "Amber." Bell Laboratories, Murray Hill, New Jersey (1985).

[22] A. Church. "A formulation of the simple theory of types." Journal of Symbolic Logic 5,1 (1940) 56-68.

[23] A. Church. "The Calculi of Lambda-Conversion." Princeton U. Press, Princeton N.J. (1941).

[24] A. Colmerauer, H. Kanoui, R. Pasero, Ph. Roussel. "Un système de communication hommemachine en francais." Rapport de recherche, Groupe Intelligence Artificielle, Faculté des Sciences de Luminy, Marscille (1973).

[25] R.L. Constable, J.L. Bates. "Proofs as Programs." Dept. of Computer Science, Cornell University. (Feb. 1983).

[26] R.L. Constable, J.L. Bates. "The Nearly Ultimate Pearl." Dept. of Computer Science, Cornell University. (Dec. 1983).

[27] Th. Coquand. "Une théorie des constructions." Thèse de troisième cycle, Université Paris VII (Jan: 85).

[28] Th. Coquand, G. Huet. "A Theory of Constructions." Preliminary version, presented at the International Symposium on Semantics of Data Types, Sophia-Antipolis (June 84).

[29] Th. Coquand, G. Huet. "Constructions: A Higher Order Proof Systern for Mechanizing Mathematics." EUROCAL85, Linz, Springer-Verlag LNCS 203 (1985).

[30] Th. Coquand, G. Huet. "Concepts Mathématiques et Informatiques Formalisés dans le Calcul des Constructions." Colloque de Logique, Orsay (Juil. 1985).

[31] Th. Coquand, G. Huct. "A Calculus of Constructions." To appear, JCSs (1986). 
[32] J. Corbin, M. Bicloit. "A Rehabititation of Robinson's Unification Algorithm." IFIP 83, Elsevier Science (1983) 909-914.

[33] G. Cousincaun, IP.L. Curien and M. Mauny. "The Categorical Abstract Machine." In Functional Programming Languages and Computer Architecture, Ed. J. P. Jonamnaud, SpringerVerling LNCS 201 (1985) 50-64.

[34] P.L. Curien. "Combinateurs catégoriques, algorithmes séquentiels et programmation applicative." Thèse de Doctorat d'Etat, Université Paris VII (Dec. 1983).

[35] P. L. Curion. "Categorical Combinatory Logic." ICALP 85, Nafplion, Springer-Verlag LNCS 194 (1985).

[36] P.L. Curien. "Catcgorical Combinators, Sequential Algorithms and Functional Programming." Pitman (1986).

[37] H. B. Curry, R. Feys. "Combinatory Logic Vol. I." North-Holland, Amsterdam (1958).

[38] D. Van Daalen. "The language theory of Automath." Ph. D. Dissertation, Technological Univ. Eindhoven (1980).

[39] Luis Damas, Robin Milner. "Principal type-schemas for functional programs." Edinburgh University (1982).

[40] P.J. Downey, R. Scthi, R. Tarjan. "Variations on the common subexpression problem." JACM $27,4(1980) 758-771$.

[41] Dummett. "Elements of Intuitionism." Clarendon Fress, Oxford (1977).

[42] F. Fages. "Tormes canoniques dans les algèbres booléennes et application à la démonstration automatique en logique de premier ordre." Thèse de 3ème cycle, Univ. de Paris VI (Juin 1983).

[43] F. Fages. "Associative-Commutative Unification." Submitted for publication (1985).

[44] F. Fages, G. Huet. "Unification and Matching in Equational Theories." CAAP 83, l'Aquila, Italy. In Springer-Vcrlag LNCS 159 (1983).

[45] P. Flajolet, J.M. Steyaert. "On the Analysis of Tree-Matching Algorithms." in Automata, Languages and Programming 7th Int. Coll., Lecture Notes in Computer Science 85 Springer Verlag (1980) 208-219.

[46] S. Fortune, D. Leivant, M. O'Donnell. "The Expressiveness of Simple and Second-Order Type Structures." Journal of the Assoc. for Comp. Mach., 30,1, (Jan. 1983) 151-185.

[47] G. Trege. "Begriffschrift, a formula language, modeled upon that of arithmetic, for pure thought." (1879). Reprinted in From Frege to Gölel, J. van Heijenoort, Harvatrd University Press, 1967.

[48] G. Gentzen. "The Collected Papers of Gerhard Gentzen." Ed. E. Szabo, North-Holland, Arnsterdam (1969). 
[40] J.Y. Cirard. "Une extension de l'interprétation di: Gödel à l'analyse, et son application à l'álinination des conpures dans l'analyse of la thécorie des tiypes. Proceedings of the Second Scandinavian Logje Symposium, Ed. J.E. Fenstiad, North Holland (1970) 63 - 92.

[50] J.Y. Girard. "Interprótation fonctionnelle et élimination des coupures dans l'arithmétique d'ordre supérieure." 'Thèse d'Etat, Universitć Paris VII (1972).

[51] K. Gödel. "Ubcr eine bisher noch nicht benutze Erweitrung des finiten Standpunktes." Dialectica, 12 (1958).

[52] W. D. Goldfarb. "The Undecidability of the Second-order Unification Problem." Theoretical Computcr Science, 13, (1981) 225-230.

[53] M. Gordon, R. Milner, C. Wadsworth. "A Metalangnage for Interactive Proof in LCF." Internal Report CSR-16-77, Department of Computer Science, University of Edinburgh (Sept. 1977).

[54] M. J. Gordon, A. J. Milner, C. P. Wadsworth. "Edinburgh LCF" Springer-Verlag LNCS 78 (1979).

[55] W. E. Gould. "A Matching Procedure for Omega Order Logic." Scientific Report 1, ATCRL 66-781, contract AF 19 (628)-3250 (1966).

[56] J. Guard. "Automated Logic for Semi-Automated Mathematics." Scientific Report 1, AFCRL (1964).

[57] J. Herbrand. "Recherches sur la théorie de la clémonstration." Thèse, U. de Paris (1030). In: Ecrits logiques de Jacques Herbrand, PUF Paris (1968).

[58] C. M. Hoffmann, M. J. O'Donnell. "Programming with Equations." ACM Transactions on Programming Languages and Systems, 4,1 (1982) 83-112.

[59] W. A. Howard. "The formula-as-types notion of construction." Unpublished manuscript (1969). Reprinted in to H. B. Curry: Essays on Combinatory Logic, Lambda Calculus and Formalism, Eds Seldin J. P. and Hindley J. R., Academic Press (1980).

[60] G. Huet. "Constrained Resolution: a Complete Method for Type Theory." Ph.D. Thesis, Jennings Computing Center Report 1117, Case Western Reserve University (1972).

[61] G. Huet. "A Mcchanization of Type Theory." Proceedings, 3rd IJCAI, Stanford (Aug. 1073).

[62] G. Huet. "The Undecidability of Unification in Third Order Logic." Information and Control 22 (1973) 257-267.

[63] G. Huet. "A Unification Algorithm for Typed Lambda Calculus." Theoretical Computer Science, 1.1 (1975) 27-57.

[64] G. Huct. "Résolution d'équations dans des langages d'ordre 1,2, .. w." Thèse d'Etat, Univcrsité Paris VII (1976).

[65] G. Huct. "Confluent Reductions: Abstract Properties and Applications to Term Rewriting Systcms." J. Assoc. Comp. Mach. 27,4 (1980) 797-821. 
[66] (r. Huct. "A Complete Proof of Correctness of the Kunth-Bendix Completion Ngorithm." JCSS 23,1 (1981) $11-21$.

[67] G. Huet. "Initiation à la Thécorie des Catégories." Polycopié de cours de DEA, Université Paris VII (Nov. 1985).

[68] G. Huet. "Cartesian Closed Categories and Lambda-Calculus." Calegory Theory Seminar, Carnegie-Mcllon University (Dec. 1985).

[69] G. Huct, J.M. Hullot. "Proofs by Induction in Equational Theories With Constructors." JACM 25,2 (1982) 239-266.

[70] G. Huet, J.J. Lévy "Call by Need Computations in Non-Ambiguous Linear Term Rewriting Systemis." Rapport Laboria 359, IRIA (Aug. 1979).

[71] G. Huet, D. Oppen. "Equations and Rewrite Rules: a Survey." In Formal Langiages: Perspectives and Open Problems, Ed. Book R., Acadenic Press (1080).

[72] J.M. Hullot "Compilation de Formes Canoniques dans les Théories Equationnelles." Thèse de 3ème cycle, U. de Paris Sud (Nov. 80).

[73] Jean Pierre Jouannaud, Helene Kirchner. "Completion of a set of rules modulo a set of equations." (April 1984).

[74] L.S. Jutting. "A translation of Landau's "Grundlagen" in AUTOMATH." Eindhoven University of Technology, Dept of Mathernatics (Oct. 1976).

[75] L.S. van Benthem Jutting. "The language theory of $\Lambda_{\infty}$, a typed $\lambda$-calculus where terms are types." Unpublished manuscript (1984).

[76] G. Kahn, G. Plotkin. "Domaines concrets." Rapport Laboria 336, IRIA (Déc. 1978).

[77] J. Ketonen, J. S. Weening. "The language of an interactive proof checker." Stanford University (1984).

[78] J. Ketonen. "EKL-A Mathematically Oriented Proof Checker." 7th Intcrnational Conference on Automated Deduction, Napa, California (May 1984). Springer-Verlag LNCS 170.

[79] J. Ketonen. "A mechanical proof of Ramsey thcorem." Stanford Univ. (1983).

[80] S.C. Kleene. "Introduction to Meta-mathematics." North Holland (1952).

[81] S.C. Kleene. "On the interpretation of intuitionistic number theory." J. Symbolic Logic 31 (1945).

[82] S.C. Kleene. "On the intorpretation of intuitionistic number theory." J. Symbolic Lugic 31 (1945).

[83] J.W. Klop. "Combinatory Reduction Systems." Ph. D. Thesis, Mathematisch Centrum Ansterdam (1980).

[84] D. Knuth, P. Bendix. "Simple word problems in universal algebras". In: Computational Problems in Abstract Algebra, J. Leech Ed., Pergamon (1970) 263-297. 
[85] D.E. Kunth, J. Morris, V. Pratt. "Past Pattern Matching in Strings." SILM Journal on Computing 6,2 (1977) $323 \times 350$.

[86] G. Kreisel. "On the interpretation of nonfinitist proofs, Part I, II." JSL $16(1952,1953)$.

[87] J. Lambek. "From Lambda-calcultns to Cartesian Closed Categorics." in 'To H. B. Curry: Essays on Combinatory Logic, Lamlda-calculus and Formalism, Eds. J. P. Scldin and J. R. Ilindley, Acadcric Press (1980).

[88] J. Lambek and P. J. Scott. "Aspects of IFigher Order Categorical Logic." Contemporary Mathematics 30 (1984) 145-174.

[89] P. J. Landin. "The next 700 progranming languages." Comm. ACM 9,3 (1966) 157-166.

[90] Plilippe Le Chenadec. "Tormes canoniques dans les algèbres finiment présentées." Thèse de 3ème cycle, Univ. d'Orsay (Juin 1983).

[91] D. Leivant. "Polymorphic type infercnce." 10th ACM Conference on Principles of Programming Languages (1983).

[92] D. Leivant. "Structural semantics for polymorphic data types." 10th ACM Conference on Principles of Programming Languages (1083).

[93] J.J. Lévy. "Réductions correctes et optimales.dans le $\lambda$-calcul." Thèse d'Etat, U. Paris VII (1978).

[04] S. MacLane. "Categories for the Working Mathematician." Springer-Verlag (1071).

[95] D. MacQueen, G. Plotkin, R. Sethi. "An ideal model for recursive polymorphic types." Proceedings, Principles of Programming Languages Symposium, Jan. 1984, 165-174.

[96] D. B. MacQueen, R. Sethi. "A semantic model of types for applicative languages." ACM Symposium on Lisp and Functional Programming (Aug. 1982).

[97] E.G. Manes. "Algebraic Theories." Springer-Verlag (1976).

[98] C. Mann. "The Connection between Equivalence of Proofs and Cartesian Closed Categories." Proc. London Math. Soc. 31 (1975) 289-310.

[99] A. Martelli, U. Montanari. "Theorem proving with structure sharing and efficient unification." Proc. 5th IJCAI, Boston, (1977) p 543.

[100] A. Martelli, U. Montanari. "An Ifficient Unification Algorithm." $\Lambda$ CM Trans. on Prog. Lang. and Syst. 4,2 (1982) 258-282.

[101] William A. Martin. "Determining the equivalence of algebraic expressions by hash coding." JACM 18,4 (1971) 549-558.

[102] P. Martin-I,öf. "A theory of types." Report 71-3, Dept. of Mathematics, University of Stockholm, Fcb. 1971, revised (Oct. 1971).

[103] P. Martin-J,öf. "About models for intuitionistic type theories and the notion of definitional cquality." Paper read at the Orléans Logic Conference (1972). 
[104] P. Martin-Löf. "An intuitionistic Theory of Types: predicative part." Logic Colloquium 73, Eds. FI. Rose and J. Sepherdson, North-Holland, (1974) 73-118.

[105] P. Martin-Löf. "Constructive Mathematics and Computer Programming." In Logic, Methodology aud Philosoply of Scicnce 6 (1980) 153-175, North-Holland.

[106] P. Martin-Löf. "Intuitionistic Type Theory." Studies in Proof Theory, Bibliopolis (1984).

[107] J. Mc Carthy. "Recursive functions of symbolic expressions and their computation by machine." CACM 3,4 (1960) 184-195.

[108] N. McCracken. "An investigation of a programming language with a polymorphic type structure." Ph.D. Dissertation, Syracuse University (1979).

[109] D.A. Miller. "Proofs in Higher-order Logic." Ph. D. Dissertation, Carnegic-Mellon University (Aug. 1983).

[110] D.A. Miller. "Expansion tree proofs and thcir conversion to natural deduction proofs." Technical report MS-CIS-84-6, University of Pennsylvania (Feb. 1984).

[111] R. Milner. "A Theory of Type Polymorphism in Programming." Journal of Computer and System Sciences 17 (1978) 348-375.

[112] R. Milner. "A proposal for Standard ML ." Report CSR-157-83, Computer Science Dept., University of Edinburgh (1983).

[113] R.P. Nederpelt. "Strong normalization in a typed $\lambda$ calculus with $\lambda$ structuned types." Ph. D. Thesis, Eindhoven University of Technology (1973).

[114] R.P. Ncderpelt. "An approach to theorem proving on the basis of a typed $\lambda$-calculus." 5 th Conference on Automated Deduction, Les Arcs, France. Springer-Verlag LNCS 87 (1980).

[115] G. Nelson, D.C. Oppen. "Fast dccision procedures based on congruence closure." JACM $27,2(1980) 356-364$.

[116] M.H.A. Newman. "On Theories with a Combinatorial Definition of "Equivalence"." Annals of Math. 43,2 (1942) 223-243.

[117] B. Nordström. "Programming in Constructive Set Theory: Some Examples." Proceedings of the ACM Conference on Functional Programming Languages and Computcr Architecture, Portmouth, New Hampshire (Oct. 1981) 141-154.

[118] B. Nordström. "Description of a Simple Programming Language." Report 1, Programming Methodology Group, University of Goteborg (Apr. 1984).

[119] B. Nordström, K. Petersson. "Types and Specifications." Information Processing 83, Ed. R. Mason, North-Holland, (1983) 915--920.

[120] B. Nordström, J. Smith. "Propositions and Specifications of Programs in Martin-Löf's Type Theory." BIT 24, (1984) 288-301.

[121] A. Obtulowicz. "The Logic of Catcgorics of Partial Functions and its Applications." Dissertationes Mathernaticae 241 (1082). 
[122] M.S. Paterson, M.N. Wegunau. "Linear Unification." J. of Computer and Systems Sciences 16 (1978) $158-167$.

[123] L. Paulson. "Recent Devclopments in LCF : Examples of structural induction." Technical Report No 34, Computer Laboratory, University of Cambridge (Jan. 1983).

[124] L. Paulson. "Tactics and Tacticals in Cambridge LCF." Technical Report No 39, Computer Laboratory, University of Cambridge (July 1983).

[125] I. Paulson. "Vcrifying the unification algorithrn in LCF." Technical report No 50, Computer Laboratory, University of Cambridge (March 1984).

[126] L. C. Paulson. "Constructing Recursion Operators in Intuitionistic Type Theory." Tech. Report 57, Computer Laboratory, University of Cambridge (Oct. 1984).

[127] G.IE. Peterson, M.E. Stickel. "Complete Sets of Reduction for Equational Theories with Complete Unification Algorithms." JACM 28,2 (1981) 233-264.

[128] T. Pietrzykowski, D.C. Jensen. "A complete mechanization of w-order type theory." Proccedings of ACM Annual Conference (107,2).

[129] T. Pietrzykowski. "A Complete Mechanization of Second-Order Type Theory." JACM 20 (1973) 333-364.

[130] D. Prawitz. "Natural Deduction." Almqist and Wiskell, Stockolm (1965).

[131] D. Prawitz. "Ideas and results in proof theory." Proceedings of the Second Scandinavian Logic Symposium (1971).

[132] I'RL staff. "Implementing Mathematics with the NUPRL Proof Development System." Computer Science Department, Cornell University (May 1985).

[133] W. V. Quine. "The problem of simplifying truth functions." Amer. Math. Monthly bf 59,8 (1952) 521-531.

[134] H. Rasiowa, R. Sikorski "The Mathematics of Metamathematics." Monografie Matematyczne tom 41, PWN, Polish Scientific Publishers, Warszawa (1963).

[135] J. C. Reynolds. "Definitional Interpreters for Higher Order Programming Languages." Proc. ACM National Conference, Boston, (Aug. 72) 717-740.

[136] J. C. Reynolds. "Towards a Theory of Type Structure." Programming Symposium, Paris. Springer Verlag LNCS 19 (1974) 408-425.

[137] J. C. Reynolds. "Types, abstraction, and parametric polymorphism." IFIP Congress'83, Paris (Sept. 1983).

[138] J. C. Reynolds. "Polymorphism is not set-theoretic." International Symposium on Semantics of Data Types, Sophia-Antipolis (June 1984).

[139] J. C. Reynolds. "Three approaches to type structure." TAPSOFT Advanced Scminar on the Role of Semantics in Software Development, Berlin (March 1985). 
[.40] J. A. Robinson. "A Machinc-Oriented Jogic Based on the Rewolution Principle." JACM 12 (1965) 32-41.

[141] J. A. Robinson. "Computational Logic: the Unification Computation." Machine Intelligence 6 Eds B. Moltzer and D.Michie, American Elsevier, New-York (1971).

[142] D. Scott. "Constructive validity." Symposium on Automatic Dcmonstration, Springer-Verlag Lecture Notes in Mathematics, 125 (1970).

[143] D. Scott. "Relating Theories of the Lambda-Calculus." in To H. B. Curry: Essays on Combinatory Logic, Lambda-calculus and Formalism, Eds. J. P. Seldin and J. R. Hindley, Academic Press (1980).

[144] J.R. Shoenfield. "Mathematical Logic." Addison-Wesley (1967).

[145] R.E. Shostak "Deciding Combinations of Theories." JACM 31,1 (1985) 1 12.

[146] J. Smith. "Course-of-values recursion on lists in intuitionistic type theory." Unpublished notes, Göteborg University (Sept. 1981).

[147] J. Smith. "The identification of propositions and types in Martin-Lof's type theory : a programming example." International Conference on Foundations of Computation Theory, Borgholm, Sweden, (Aug. 1983) Springer-Verlag LNCS 158.

[148] R. Statman. "Intuitionistic Propositional Logic is Polynomial-space Complete." Theoretical Computer Science 9 (1979) 67-72, North-Holland.

[149] R. Statman. "The typed Lambda-Calculus is not Elementary Recursive." Thcoretical Computer Science 9 (1979) 73-81.

[150] S. Stenlund. "Combinators $\lambda$-terms, and proof theory." Reidel (1972).

[151] M.E. Stickel "A Complete Unification Algorithm for Associative-Commutative Functions." JACM 28,3 (1981) 423-434.

[152] M.E. Szabo. "Algebra of Proofs." North-Holland (1978).

[153] W. Tait. "A non constructive proof of Gentzen's Hauptsatz for second order predicate logic." Bull. Amer. Math. Soc. 72 (1966).

[154] W. Tait. "Intensional interpretations of functionals of finite type I." J. of Symbolic Logic 32 (1967) 198-212.

[155] W. Tait. "A Realizability Interpretation of the Theory of Species." Logic Colloquium, Ed. R. Parikh, Springer Verlag Lecture Notes 453 (1975).

[156] M. Takalashi. "A proof of cut-elimination theorem in simple type theory." 'J. Math. Soc. Japan 19 (1967).

[157] G. Takeuti. "On a generalized logic calculus." Japan J. Math. 23 (1953).

[158] G. Takenti. "Proof theory." Studies in Logic 81 Amsterdam (1975). 
[159] R.. E. Tarjan. "Efficiency of a good but non linew set union algorithm." JACM 22,2 (1975) 2.15225.

[160) R. F. Tarjan, J. van Lecuwen. "Worst-case Analysis of Set Union Algorithms." JACM 31,2 (1985) $245 \cdots 281$.

[161] A. Tarski. "A lattice-thcorctical fixpoint theorcm and its applications." Pacific J. Math. 5 (1955) 285309 .

[162] D.A. Turner. "Miranda: A non-strict functional language with poly morphic types." In Functional Programming Languages and Computer Architecture, Ed. J. P. Jouammaud, SpringerVerlag LNCS 201 (1985) 1-16.

[163] R. de Vrijer "Big Trees in a $\lambda$-calculus with $\lambda$-expressions as types." Conference on $\lambda$-calculus and Computer Science Theory, Rome, Springer-Verlag LNCS 37 (1975) 252-271.

[164] D. Warren "Applied Logic - Its use and implenentation as a programming tool." Ph.D. Thesis, University of Edinburgh (1977). 
